\title{
Transcriptomic analysis of a moderately growing subisolate Botryococcus braunii 779 (Chlorophyta) in response to nitrogen deprivation
}

Lei Fang ${ }^{1,6 \dagger}$, Deying Sun ${ }^{2,7 \dagger}$, Zhenyu Xu ${ }^{1}$, Jing He ${ }^{3}$, Shuyuan $\mathrm{Qi}^{1}$, Xin Chen ${ }^{4}$, Wee $\mathrm{Chew}^{5}$ and Jianhua Liu ${ }^{1,3^{*}}$

\begin{abstract}
Background: The colonial microalga Botryococcus braunii has been brought to people's attention for its conspicuous ability to accumulate a variety of lipids including hydrocarbons. B. braunii strains are classified into 3 races based on the types of hydrocarbons. A and B races are known to accumulate high level of lipids. However, their extreme slow growth rate has impeded its application for renewable biofuel production.

Results: In this study, we report the transcriptomic response of a moderately growing subisolate from the culture of Botryococcus sp. CCALA-779 upon nitrogen deprivation (ND). We show that the subisolate has an average growth rate of $0.52 \mathrm{~g} \mathrm{l}^{-1}$ day $^{-1}$ under photoautotrophic growth conditions and lipid content is enhanced to $75 \%$ of CDW upon ND. Both rDNA sequence and hydrocarbon composition analyses indicate that the subisolate belongs to $A$ race $B$. braunii. Hence, it is designated as $B$. braunii 779 . We show that $B$. braunii 779 transcriptome shares homology to majority of the $A$ race but not $B$ race $B$. braunii ESTs, suggesting that transcriptomes of $A$ race differ from that of $B$ race. We found that many homologous ESTs between A races 779 and Bot- 88 are unknown sequences, implying that $A$ race contains many unknown genes. Pathway-based transcriptomic analysis indicates that energy metabolisms are among the top expressed functions in log-phase cells, indicating that the slow growth rate is a result that energy flow is directed to lipid biosynthesis but not population growth. Upon ND, reconfiguration of metabolisms for reducing power is apparent, suggesting that $B$. braunii 779 is rapidly adapting under ND condition by transcriptomic reprogramming.
\end{abstract}

Conclusions: Taken together, our result shows that the subisolate B. braunii 779, similar to the Gottingen strain, is useful for biofuel production. Difference between transcriptomes of $A$ and $B$ races implies that different races of $B$. braunii strains belong to different sub-species. Furthermore, there are many novel genes that are unique to $A$ race, suggesting that sequences of many enzymes involved in hydrocarbon biosynthesis are not currently known. We propose that $B$. braunii transcriptomes provide a rich source for discovery of novel genes involved in hydrocarbon biosynthesis.

Keywords: Botryococcus braunii, Green microalgae, Hydrocarbon, Transcriptome, Response to nitrogen deprivation

\footnotetext{
*Correspondence: liujh2013@zju.edu.cn

${ }^{\dagger}$ Lei Fang and Deying Sun contributed equally to this work

${ }^{3}$ Ocean Research Centre of Zhoushan, Zhejiang University, 10 Tiyu Road,

Room 502, Zhoushan 316021, Zhejiang, China

Full list of author information is available at the end of the article
} 


\section{Background}

Microalgae have been getting renewed interests for its $\mathrm{CO}_{2}$ mitigation and lipid production. Photosynthetic microalgae, like green plants, utilize photon energy to convert $\mathrm{CO}_{2}$ (in presence of water) into sugar and oxygen. Lipid yield from oleaginous algae is believed to be at least an order of magnitude higher than that of many energy plants $[1,2]$, besides that cultivation of microalgae can avoid competing arable lands and irrigation water for crops [3, 4]. Many oleaginous microalgae accumulate triacylglycerols (or TAG) that need to be transesterified with methanol to methyl esters of fatty acids and glycerol, for example, before being refined to biodiesel [5]. On the other hand, oleaginous green colonial microalga Botryococcus braunii has been brought to people's attention for its conspicuous ability to accumulate a variety of lipids including hydrocarbons that are found in petroleum deposits and can be refined directly without the need of esterification because they are not fatty acids $[4,6]$.

However, B. braunii has suffered from an extreme slow growth (i.e., population doubling time is approximately 5-7 days) that has hampered from its application for biofuel production [6]. The slow growth has also prevented it from genetic studies. Hence, many researches have focused on the analysis of hydrocarbon compositions and the discovery of DNA sequences encoding enzymes involved in biosynthesis of hydrocarbons [7-12]. Depending on the types of hydrocarbons accumulated in cells and cell wall matrix, $B$. braunii is classified into three principal races, $\mathrm{A}, \mathrm{B}$, and $\mathrm{L}$. A race produces very long chain fatty acid (or VLCFA)-derived odd-carbon-numbered $n$-alkadiene and alkatriene, B race produces mainly botryococcenes (or triterpenoids), and $\mathrm{L}$ race produces lycopadiene (or tetraterpenoid) [13-16]. Several genes encoding enzymes such as triterpene methyltransferases TMTs [8], 1-deoxy-D-xylulose 5-phosphate synthase DXS [10], squalene synthase-like SSL botryococcenes synthase [9, 17], Botryococcus squalene synthase BSS [18], and squalene epoxidases SQE [11] have been cloned and their enzymatic activities have been tested.

Genomic sequences of a number of photosynthetic microalgae have been completed (http://genome.jgi-psf. org). These comprehensively annotated genomes have provided rich sources for annotation of novel genomes and transcriptomes. Using the comprehensively annotated genomes, de novo transcriptome analysis without reference genomic sequences has permitted the discovery of genes and reconstitution of metabolic pathways in some non-model microalgae [19-22]. De novo transcriptomic analyses in B. braunii have extended our knowledge on potential enzymes involved in biosynthesis of hydrocarbons in B. braunii [21, 23-25]. For example,
19 out of 55 sequences encoding enzymes potentially involved in biosynthesis of VLCFA have been discovered in A race B. braunii Bot-88 [24] and 100 curated and machine-assembled sequences potentially involved in biosynthesis of botryococcenes and squalene have been revealed in B race B. braunii Showa [21]. However, it is unclear why not all the enzymes that are involved in biosynthesis of VLCFA are found in the transcriptomes of A race $B$. braunii.

ND is one of the most widely used methods for enhancement of storage lipids in a number of TAG-containing green microalgae $[2,5,22]$. In A race B. braunii, nitrogen limitation enhances accumulation of oleic acid [26], but not hydrocarbons [27]. Global transcriptional profiling of microalgal cells in response to ND using nextgeneration sequencing (or NGS) technologies allows identification of gene regulatory networks involved in adaptation and survival $[22,28,29]$.

In this study, we report (1) the characterization of a moderately growing subisolate derived from the Botryococcus sp. CCALA779 culture, (2) biochemical analysis of the lipids and hydrocarbons accumulated in the subisolate, and (3) transcriptomic profiling of the subisolate in response to ND. We show that the subisolate can reach a maximal growth rate of $1.23 \mathrm{~g} \mathrm{l}^{-1} \mathrm{~d}^{-1}$ and an average growth rate of $0.52 \mathrm{~g} \mathrm{l}^{-1} \mathrm{~d}^{-1}$ in $2 \times \mathrm{BB}$ medium [30] under photoautotrophic growth conditions. Lipid contents are enhanced by nitrogen deprivation (ND). The 18S rRNA sequence-based phylogenetic analysis places the subisolate to the group of A race. Consistent with this, GC-MS analysis of hydrocarbon composition in the subisolate reveals the presence of heptacosdiene and heptacostriene. Four giga-nucleotide short-read sequences of RNA (or cDNA) generated using Illumina sequencing platform were assembled by Trinity software. Approximately $20 \%$ (i.e., 12,292) of the non-redundant transcriptome was found to have best-hit in the 6 algal genomes that are comprehensively annotated (http://genome.jgi-psf.org). Comparison to other previously reported transcriptomes of other $B$. braunii strains implies that the similarity between transcriptomes of A and B races is very low (i.e., at a level of $15 \%)$. On the other hand, majority of the homologous ESTs between A races are unknown sequences. Taken together, our results indicate that the moderately growing subisolate belong to the $\mathrm{A}$ race $B$. braunii. Transcriptomes between A and B races B. braunii differ dramatically, suggesting that $A$ and $B$ races belong to different sub-species. Additionally, many ESTs in $A$ race transcriptomes are unknown sequences, providing a useful resource for discovery of novel genes. We propose that the B. braunii 779 is similar to other 
well-characterized strains such as Gottingen strain [27], attractive for biofuel production.

\section{Results}

Accumulation of lipid and hydrocarbon in a moderate growing Botryococcus subisolate is enhanced by ND

A moderately growing subisolate from a culture of Botryococcus sp. CCALA 779 on solid BB medium was obtained. Photoautotrophic growth rate of the subisolate in liquid BB medium was examined under the condition of $250 \mu \mathrm{mol}$ photon $\mathrm{m}^{-2} \mathrm{~s}^{-1}$. The growth curve indicated that this subisolate exhibited a maximum growth rate of $0.61 \mathrm{~g} \mathrm{l}^{-1} \mathrm{~d}^{-1}$ (i.e., gram of cell dry weight or CDW per liter per day) and a maximum cell density of $1.98 \mathrm{~g} \mathrm{l}^{-1}$ in terms of CDW per liter (Fig. 1a; Table 1). When using $2 \times$ $\mathrm{BB}$ medium (i.e., to double the nutrient concentration of the $\mathrm{BB}$ medium), we found that both the growth rate and cell maximum density were doubled to $1.23 \mathrm{~g} \mathrm{l}^{-1} \mathrm{~d}^{-1}$ and $4 \mathrm{~g} \mathrm{l}^{-1}$, respectively. Subsequently, we examined the content of lipid and hydrocarbon in log-phase cells (e.g., at the density of $\sim 1.61 \mathrm{~g} / \mathrm{L}$ in $1 \times \mathrm{BB}$ medium) that were extracted by methanol/chloroform solution (i.e., the ratio was 2:1 by volume) and hexane, respectively, using gravimetric methodologies (see "Methods"). Our results indicated that the total lipid and hydrocarbon contents in log-phase cells were approximately $0.58 \mathrm{~g} / \mathrm{L}$ (or $36 \%$ of CDW) and $0.31 \mathrm{~g} / \mathrm{L}$ (or $19 \%$ of CDW), respectively (Table 2).

To investigate whether ND treatment would enhance the lipid and/or hydrocarbon content, the log-phase cells were subjected to ND by replacing BB-N medium (or nitrogen-less $\mathrm{BB}$ medium) without altering the cell density. Three days after ND, cell density was slightly increased by $4 \%$ of CDW (i.e., from 1.61 to $1.68 \mathrm{~g} / \mathrm{L}$ ). Nile red is a fluorescent hydrophobic probe that is commonly used for detection of cellular neutral lipid and hydrocarbon deposits [31]. Fluorescence microscopic analysis of Nile red-stained subisolate cells showed that many tiny lipid deposits were visible in cells prior to ND, which were likely composed of triacylglycerols or hydrocarbons or both (Fig. 1b). On the other hand, these tiny deposits were aggregated to form much large lipid droplets upon ND. This result suggests that contents of lipids (i.e., triacylglycerols or hydrocarbons or both) are enhanced by ND in this subisolate.

Botryococcus cells tended to form bundles, which made it unsuitable for FACS analysis to determine lipid contents in individual cells. Hence, we applied gravimetric method to estimate total lipid and hydrocarbon contents in cells prior to and after ND (see "Methods"). To this end, total lipid (i.e., extracted by methanol/chloroform) and hydrocarbon (i.e., extracted by hexane) contents were found to increase by 2.2 -fold and 2.8 -fold,

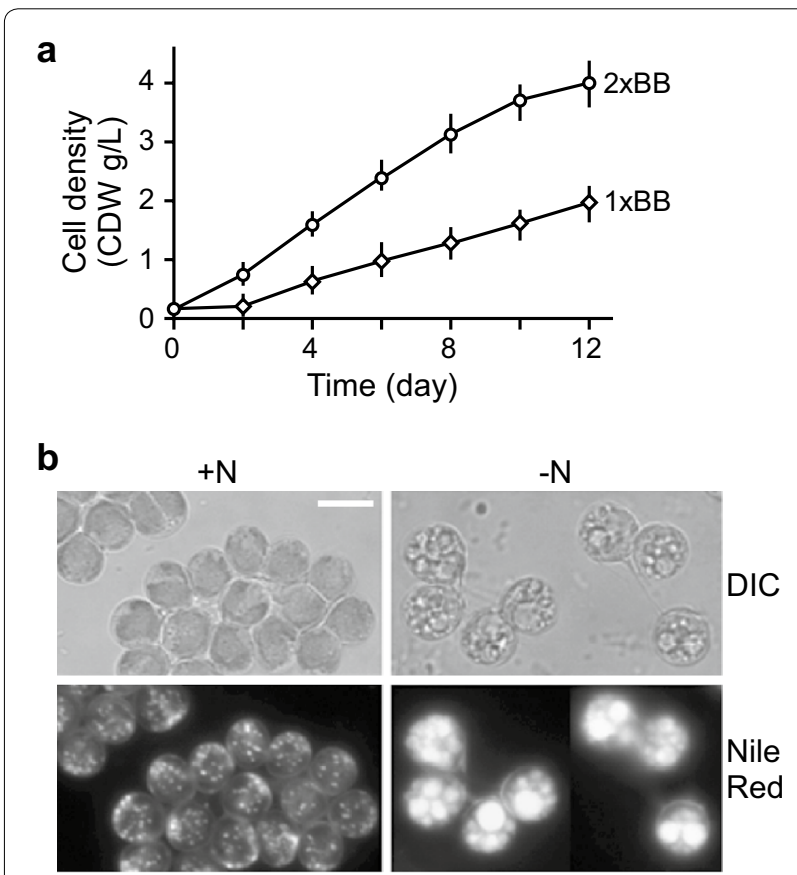

C

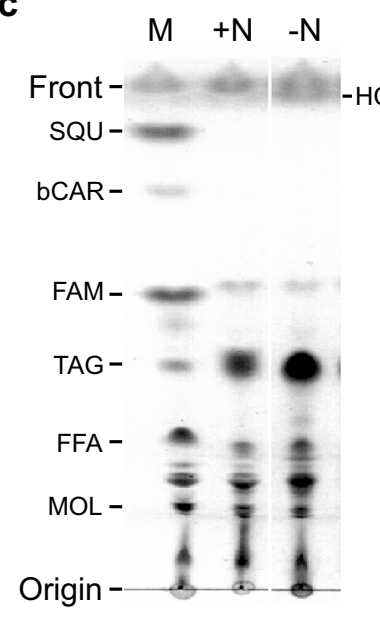

Fig. 1 Characterization of the moderately growing subisolate from a Botryococcus CCALA 779 culture. a Growth curve of the subisolate. $X$ - and $Y$-axis indicate time in days and cell dry weight in gram per liter. Nutrient strength of $1 \times B B$ and $2 \times B B$ indicates medium with standard nutrient concentration and twofold of standard concentration. $\mathbf{b}$ Fluorescence microscopic analysis of Nile red-stained cells of subisolate. Cells prior to and after ND are indicated by $+\mathrm{N}$ and $-\mathrm{N}$, respectively. Cell images are captured under the setting of differential interference contrast (DIC) and fluorescence (Nile red). A scale bar of $20 \mu \mathrm{m}$ is shown. c Analysis of total lipids extracted by methanol/ chloroform solution (i.e., 2:1 by volume). Equal amount of total lipid extract from cells prior to $(+\mathrm{N})$ and after $(-\mathrm{N}) \mathrm{ND}$ is loaded onto a TLC plate and developed with hexane/diethyl ether/acetic acid solution (i.e., 35:15:0.1 by volume). Origin and front of TLC are indicated. Marker (M) of standard chemicals includes squalene (SQU), betacarotene (bCAR), FAME $\mathrm{C}_{14}-\mathrm{C}_{22}$ (FAM), tri-oleic acid glyceride (TAG), oleic acid (FFA), and mono-olein (MOL). HC hydrocarbon. 
Table 1 Growth curves of the Botryococcus sp. subisolate

\begin{tabular}{|c|c|c|c|c|c|}
\hline Time & $\mathrm{CDW} 1^{\mathrm{a}}$ & & CDW2 & CDW3 & CDW (Ave \pm SD) ${ }^{b}$ \\
\hline \multicolumn{6}{|l|}{ (A) In BB medium } \\
\hline $0(d)$ & 0.140 & & 0.155 & 0.155 & $0.150 \pm 0.009$ \\
\hline 2 & 0.215 & & 0.245 & 0.235 & $0.232 \pm 0.015$ \\
\hline 4 & 0.600 & & 0.640 & 0.630 & $0.623 \pm 0.021$ \\
\hline 6 & 0.920 & & 0.960 & 0.970 & $0.950 \pm 0.026$ \\
\hline 8 & 1.150 & & 1.350 & 1.300 & $1.267 \pm 0.104$ \\
\hline 10 & 1.500 & & 1.700 & 1.650 & $1.617 \pm 0.104$ \\
\hline 12 & 1.900 & & 2.200 & 1.900 & $2.000 \pm 0.173$ \\
\hline Period & & Rate $1^{c}$ & Rate2 & Rate3 & Rate (Ave \pm SD) \\
\hline \multicolumn{6}{|l|}{ (A) In BB medium } \\
\hline $0-2$ & & 0.239 & 0.257 & 0.231 & $0.243 \pm 0.013$ \\
\hline $2-4$ & & 0.671 & 0.616 & 0.637 & $0.641 \pm 0.027$ \\
\hline $4-6$ & & 0.238 & 0.225 & 0.241 & $0.235 \pm 0.009$ \\
\hline $6-8$ & & 0.118 & 0.186 & 0.158 & $0.154 \pm 0.034$ \\
\hline Average growth rate & & 0.318 & & & \\
\hline Maximum growth rate & & 0.641 & & & \\
\hline Time & CDW1 & & CDW2 & CDW3 & CDW (Ave \pm SD) \\
\hline \multicolumn{6}{|l|}{ (B) In $2 \times$ BB medium } \\
\hline $0(d)$ & 0.140 & & 0.155 & 0.155 & $0.150 \pm 0.009$ \\
\hline 2 & 0.730 & & 0.755 & 0.750 & $0.745 \pm 0.013$ \\
\hline 4 & 1.610 & & 1.580 & 1.630 & $1.607 \pm 0.025$ \\
\hline 6 & 2.250 & & 2.500 & 2.550 & $2.433 \pm 0.161$ \\
\hline 8 & 3.000 & & 3.000 & 3.300 & $3.100 \pm 0.173$ \\
\hline 10 & 3.500 & & 3.850 & 3.850 & $3.733 \pm 0.202$ \\
\hline 12 & 3.800 & & 4.300 & 4.050 & $4.050 \pm 0.250$ \\
\hline Period & & Rate1 & Rate2 & Rate3 & Rate (Ave \pm SD) \\
\hline \multicolumn{6}{|l|}{ (B) In $2 \times$ BB medium } \\
\hline $0-2$ & & 1.2835 & 1.2070 & 1.1997 & $1.2301 \pm 0.0464$ \\
\hline $2-4$ & & 0.4851 & 0.4466 & 0.4742 & $0.4686 \pm 0.0198$ \\
\hline $4-6$ & & 0.1822 & 0.2579 & 0.2508 & $0.2303 \pm 0.0418$ \\
\hline $6-8$ & & 0.1547 & 0.0954 & 0.1376 & $0.1292 \pm 0.0305$ \\
\hline Average growth rate & & 0.515 & & & \\
\hline Maximum growth rate & & 1.230 & & & \\
\hline
\end{tabular}

a CDW stands for cell try weight, repeat number is indicated.

b Ave and SD stand for average and standard deviation based on 3 repeats.

c Growth rate in an 2 days-period of $0-2$ days, 2-4 days, 4-6 days, and 6-8 days was calculated using the formula $\sqrt[2]{C D W_{t 2} / C D W_{t 1}}-1$.

respectively (see Table 2). This result indicated that the lipid and hydrocarbon contents in the subisolate were highly enhanced upon ND.

The methanol/chloroform extract or total lipid was subsequently subjected to thin-layer chromatographic analysis. Our results indicated that levels of triacylglyceride (TAG), diacylglyceride (DAG), free fatty acid (FFA), but not monoacylglyceride (MAG) were increased in the subisolate upon ND (Fig. 1c). These results indicated that the moderately growing subisolate's neutral lipid accumulation was enhanced by ND.

\section{The subisolate belongs to the group of race $A B$. braunii}

To investigate the phylogenic placement of this subisolate, we performed the $18 \mathrm{~S}$ rDNA sequences-based phylogenetic analysis. For this reason, the 18S rDNA sequences were amplified using PCR with sequence-specific primers [32] (see "Methods"). Our result indicated 
Table 2 Lipid and hydrocarbon contents are enhanced upon nitrogen deprivation in a subisolate of Botryococcus sp.

\begin{tabular}{|c|c|c|c|c|c|}
\hline \multirow{2}{*}{$\frac{\mathrm{N} \text {-replete }}{\mathrm{CDW}(\mathrm{g} / \mathrm{L})}$} & \multicolumn{3}{|c|}{ Individual measurements } & \multirow{2}{*}{$\begin{array}{l}\text { Average } \\
1.61 \pm 0.03\end{array}$} & \multirow[t]{2}{*}{$\% \mathrm{CDW}^{\mathrm{a}}$} \\
\hline & 1.61 & 1.58 & 1.63 & & \\
\hline $\mathrm{TL}(\mathrm{g} / \mathrm{L})$ & 0.59 & 0.6 & 0.56 & $0.58 \pm 0.02$ & 36.0 \\
\hline $\mathrm{HC}(\mathrm{g} / \mathrm{L})$ & 0.30 & 0.36 & 0.28 & $0.31 \pm 0.04$ & 18.3 \\
\hline $\mathrm{N}$-depleted & \multicolumn{3}{|c|}{ Individual measurements } & Average & $\% \mathrm{CDW}$ \\
\hline CDW (g/L) & 1.67 & 1.67 & 1.70 & $1.68 \pm 0.02$ & \\
\hline $\mathrm{TL}(\mathrm{g} / \mathrm{L})$ & 1.25 & 1.29 & 1.23 & $1.25 \pm 0.03$ & 74.4 \\
\hline $\mathrm{HC}(\mathrm{g} / \mathrm{L})$ & 0.87 & 0.84 & 0.92 & $0.87 \pm 0.04$ & 51.8 \\
\hline
\end{tabular}

a CDW for cell dry weight.

that the subisolate was mostly related to the group of A race $B$. braunii spp. (Fig. 2a). It is known that A race $B$. braunii accumulates mainly the $n$-alkadiene and alkatriene [13], unlike B and L races of B. braunii that accumulate largely botryococcenes (or triterpenoids) and lycopadiene (or tetraterpenoids), respectively $[14,15]$.

To investigate whether the subisolate contained $n$-alkadiene and triene, the hexane extract was subjected to GC-MS analysis. To this end, heptacosdiene $\left(\mathrm{C}_{27} \mathrm{H}_{52}\right)$ and heptacostriene $\left(\mathrm{C}_{27} \mathrm{H}_{50}\right)$ were found to be the predominant composition in the hydrocarbon extract (Fig. 2b, c), supporting the $18 \mathrm{~S}$ rDNA sequence-based phylogenetic analysis. Hence, we designated this subisolate as A race $B$. braunii 779 . We found that the contents of total hydrocarbons (see Table 2), possibly including heptacosdiene and heptacostriene were increased upon ND in B. braunii 779.

\section{The de novo-assembled transcriptome of $B$. braunii 779 appears to mostly resemble that of Coccomyxa subellipsoidea}

To investigate the transcriptomic change upon ND, we first wanted to assemble the $B$. braunii 779 transcriptome. For this reason, B. braunii 779 log-phase growing cells prior to and 3 days after ND were collected for total RNA extraction (see "Methods"). The resulting total RNA samples were individually subjected to sequencing analysis using the Illumina HiSEQ 2000 platform (BGI). Over 27 million good reads with $90 \mathrm{nt}$ in length were obtained from each sample. All 55 million reads were pooled for de novo assembly of transcriptome using Trinity software [33] (see "Methods").

We obtained 61,220 non-redundant ESTs based on the cutoff of identity $<90 \%$ (i.e., based on CD-Hit analysis), EST length $>300 \mathrm{bps}$, and read count per EST $>40$. Annotation of the non-redundant ESTs was performed based on the best-hit proteins/genes generated by sequence homology comparison using the Basic Local Alignment
Search Tool BLASTX suite (http://blast.ncbi.nlm.nih. gov) against the "best" proteins/genes in the comprehensively annotated genomes of 5 green microalgae Coccomyxa subellipsoidea [34], Chlorella variabilis [35], Chlamydomonas reinhardtii [36], Micromonas pusilla [37], and Ostreococcus lucimarinus [38] and a brown alga Thalassiosira pseudonana [39] with a cutoff expectation value of 1E-07 (see "Methods"). To this end, a total of $12,292(20.1 \%)$ of the non-redundant ESTs in B. braunii 779 were shown to have best-hits (i.e., e value $<1.0 \mathrm{E}-07$ ) (for a complete list of annotated ESTs, see Additional file 1: Table S1). We found that majority ( $70 \%)$ of the best-hits in B. braunii 779 were derived from C. subellipsoidea, suggesting that the transcriptome of $B$. braunii 779 is most closely related to that of $C$. subellipsoidea (Fig. 3a). This observation was supported by the result of the $18 \mathrm{~S}$ rDNA sequence-based phylogenetic analysis (see Fig. 2a).

The 12,292 annotated ESTs in the transcriptome of $B$. braunii 779 were subjected to functional analysis in this study. The length of these ESTs was ranged from $301 \mathrm{bps}$ to $8,268 \mathrm{bps}$ with the median length of $663 \mathrm{bps}$ (Fig. 3b). The read count per EST was ranged from 42 to 182,192 with the median of 174 . Of the 12,292 ESTs, 8,888 were found to be associated with at least one gene-ontology (GO) function. A total of $539 \mathrm{GO}$ functions in Biological Process, 171 functions in Cellular Component, and 1,549 functions in Molecular Function associated to the B. braunii 779 transcriptome (for a complete list of ESTassociated GO functions, see Additional file 2: Table S2). Ten GO biological processes associated with the most number of ESTs are listed in Fig. 3d (for top 10 CC and MF, see Additional file 3: Figure S1).

Subsequently we found that 3,386 out of the 12,292 ESTs were associated with at least one KEGG ortholog. The metabolic pathways associated with the most number of ESTs are shown in Fig. 3e (for a complete list of EST-associated pathways, see Additional file 4: Table S3). 


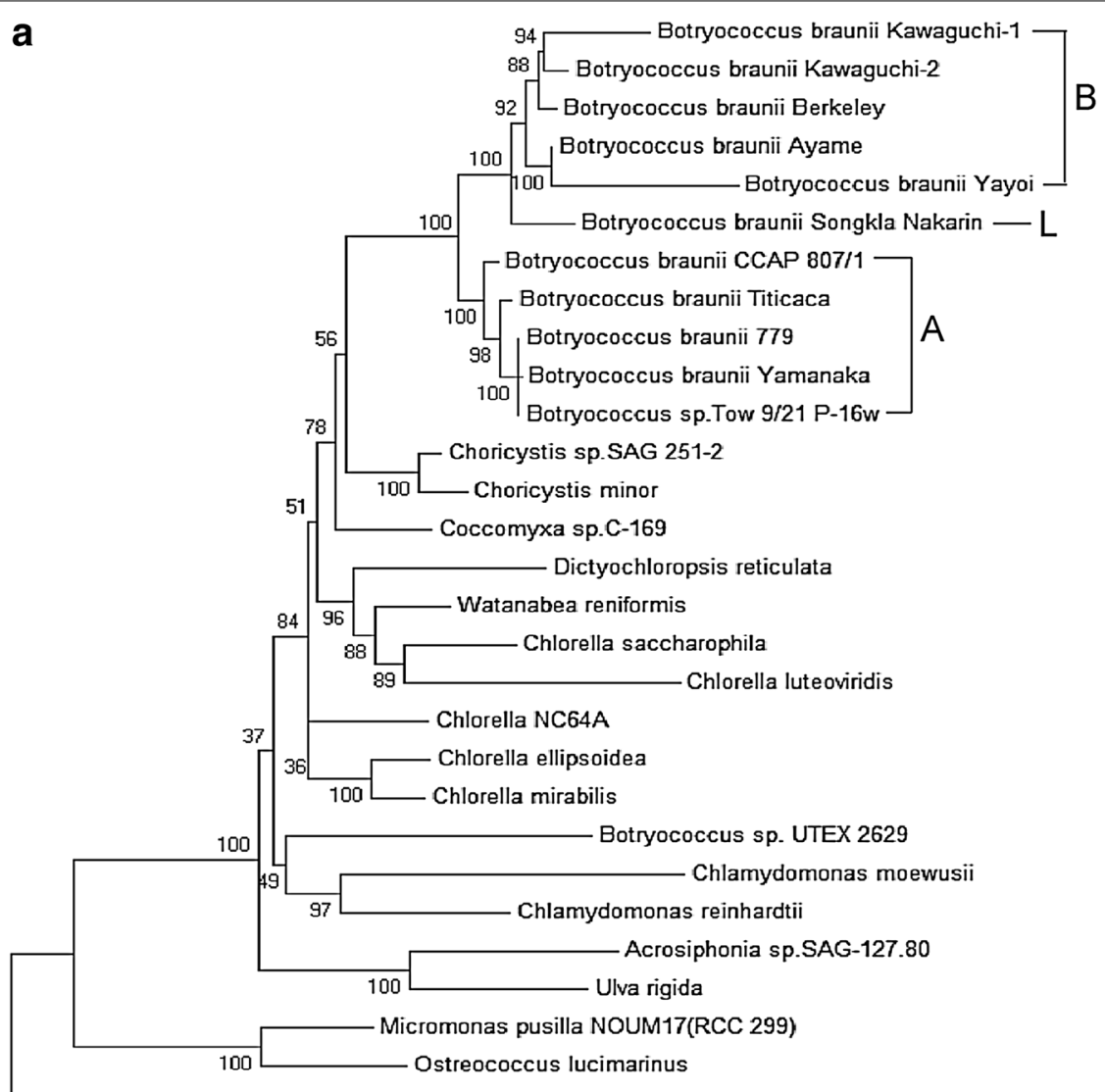

Thalassiosira pseudonana CCMP 1335

$\longmapsto .02$
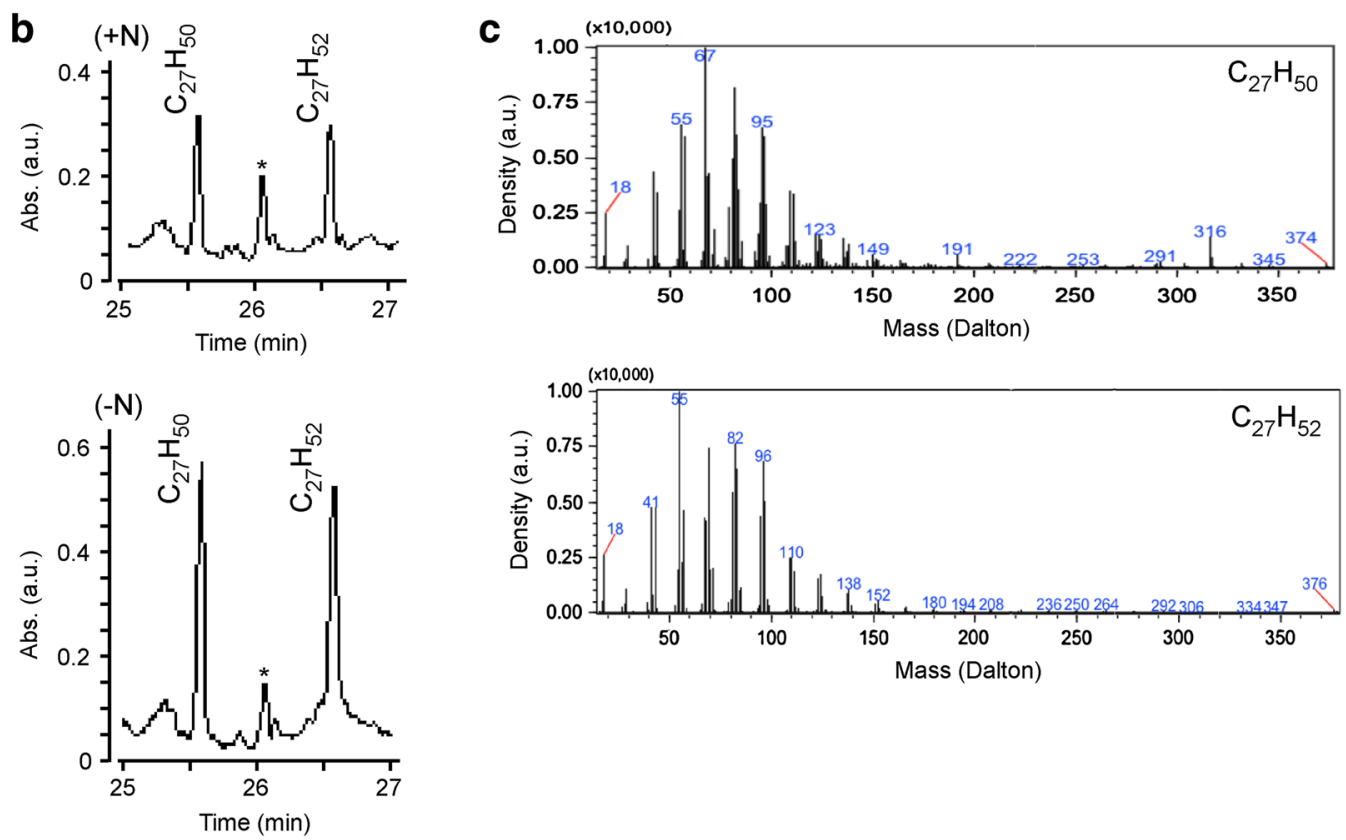
(See figure on previous page.)

Fig. 2 The subisolate belongs to the group of Botryococcus braunii race A. a Phylogenetic analysis of the subisolate with other Botryococcus spp. based on the $18 \mathrm{~S}$ rDNA sequence. Groups of Botryococcus spp. race A, B, L are indicated. The subisolate of CCALA 779 in the phylogenetic tree is indicated. $\mathbf{b}$ GC spectrum of hydrocarbons extracted from cells of the subisolate prior to and after ND. X-and Y-axis indicate time in minute (min) and absorbance (abs) in arbitrary unit (a.u.). Compositions of hydrocarbon are indicated. The asterisk $\left(^{*}\right)$ indicates an unknown chemical species. c Mass spectra of the heptacosdiene $\mathrm{C}_{27} \mathrm{H}_{52}$ and heptacostriene $\mathrm{C}_{27} \mathrm{H}_{50}$. X- and $\mathrm{Y}$-axis indicate mass in Dalton and density in arbitrary unit (a.u.), respectively.

Richness of the GO and KO (KEGG ortholog) annotation of the transcriptome allowed performing pathway-based transcriptional analysis (see "Methods").

\section{Majority of the ESTs in the previously reported A race, but not $B$ race transcriptome of $B$. braunii are found in the transcriptome of B. braunii 779}

To compare the similarity of the B. braunii 779 transcriptome with the previously reported transcriptomes of $A$ race $B$. braunii Bot- 88 and $B$ race $B$. braunii Showas, Bot70, and Bot-22, we first processed the datasets as did for the B. braunii 779 by removing any ESTs/cDNAs/contigs (for consistency, we use ESTs) whose length was less than 300nts and CD-Hit identity was $90 \%$ or greater in the transcriptomes of B. braunii Bot-88, Showa, Bot-70, and Bot-22 [21, 23-25, 35]. To this end, we obtained sets of $8,302,38,920,1,665$, and 6,848 ESTs in transcriptomes of B. braunii Bot- 88 , Showa, Bot-70, and Bot-22, respectively. The processed transcriptomes were subjected to BLASTN analysis (http://blast.ncib.nlm.nih.gov) against the transcriptome of B. braunii 779. Based on a cutoff of e value $1 \mathrm{E}-05$, we found that, compared to others, the $\mathrm{A}$ race $B$. braunii Bot- 88 transcriptome shared the highest percentage of ESTs to that of B. braunii 779 (Fig. 4a, for a complete list of homologous ESTs, see Additional file 5: Tables S4-S7).

Among the 3 published B race B. braunii transcriptomes, Showa had the most completed transcriptome $[21,23,25]$. Hence, the Showa transcriptome was used as reference for $B$ race $B$. braunii transcriptome. Sequences of B. braunii Bot- 88 , Bot-22, Bot-70, and 779 transcriptomes were subjected to homology analysis using BLASTN against that of B. braunii Showa. With a cutoff of e value $1 \mathrm{E}-05$, we found that, among others, the $B$ race $B$. braunii Bot -70 shared the highest percentage of ESTs to that of B. braunii Showa (Fig. 4b, for a complete list of homologous ESTs, see Additional file 6: Tables S8S11). It was noted that majority (i.e., $\sim 75 \%$ ) of transcriptomes between B. braunii A race 779 and B race Showa were found to be different, implying that $A$ and $B$ races belong to different sub-species of $B$. braunii.

Of the 5,860 B. braunii 779 ESTs that shared homolog to A race B. braunii Bot- 88 , we noted that only 715 (or $12.2 \%$ ) had best-hit in the "best" proteins of the
6 comprehensively annotated genomes (Fig. 4c). On the other hand, $40-60 \%$ of the B. braunii 779 sheared homologous ESTs to the B races appeared to have functional annotations. This result implied that many ESTs in B. braunii 779 or Bot- 88 without a best-hit in the 6 selected genomes might represent novel genes specific to A race $B$. braunii species.

\section{ESTs associated with energy metabolisms are abundantly expressed in the log-phase $B$. braunii 779 cells}

Individual EST levels were normalized to FPKM (i.e., Fragments Per Kilobase of EST per Million fragments mapped) and averaged from two biological repeated experiments for functional analysis of the transcriptome. Based on association with GO functions, we found that, of the top 30 most abundant ESTs ranked by level, 21 were associated with photosynthesis function (or light harvesting, GO:0009765) that was greatly enriched (i.e., 239 -fold increase, $p$ value $<1 \mathrm{E}-26$ ). Besides, glycolysis (i.e., GO:0006096) and translation (i.e., GO:0006412) were also enriched by 20 -fold and sixfold (i.e., $p$ value $=4.3 \mathrm{E}-03$ and $8.7 \mathrm{E}-03$ ), respectively (Table 3 ).

We assumed that a subset of ESTs in a pathway displaying a similar expression level (i.e., based on ranks) or expression coherence was likely to be co-regulated by a common transcriptional regulatory network. To search for potentially co-regulated transcriptional networks, we performed the sliding window analysis using a window size of 1024 ESTs and a moving step of 512 ESTs along the sorted ESTs based on rank by level (see "Methods"). According to $44 \mathrm{GO}$ biological processes and 59 KEGG metabolic pathways (i.e., number of process- or pathway-associated EST $>30$ ), we found that 2 biological processes (i.e., photosynthesis and translation) and 5 metabolic pathways (i.e., TCA cycle, glycolysis/gluconeogenesis, pentose phosphate pathway, pyruvate metabolism, and carbon fixation) whose ESTs were enriched in 1 of the 23 windows alone the sorted ESTs based on rank by level (Fig. 5). Photosynthesis and translation processes and glycolysis/gluconeogenesis, pyruvate metabolism and carbon fixation pathways were enriched in the window containing the highest ranked ESTs or in window 1 (i.e., fold change $>2, p$ value $<0.05$ after Bonferroni correction $[40,41])$. Similar to translation, ribosome 

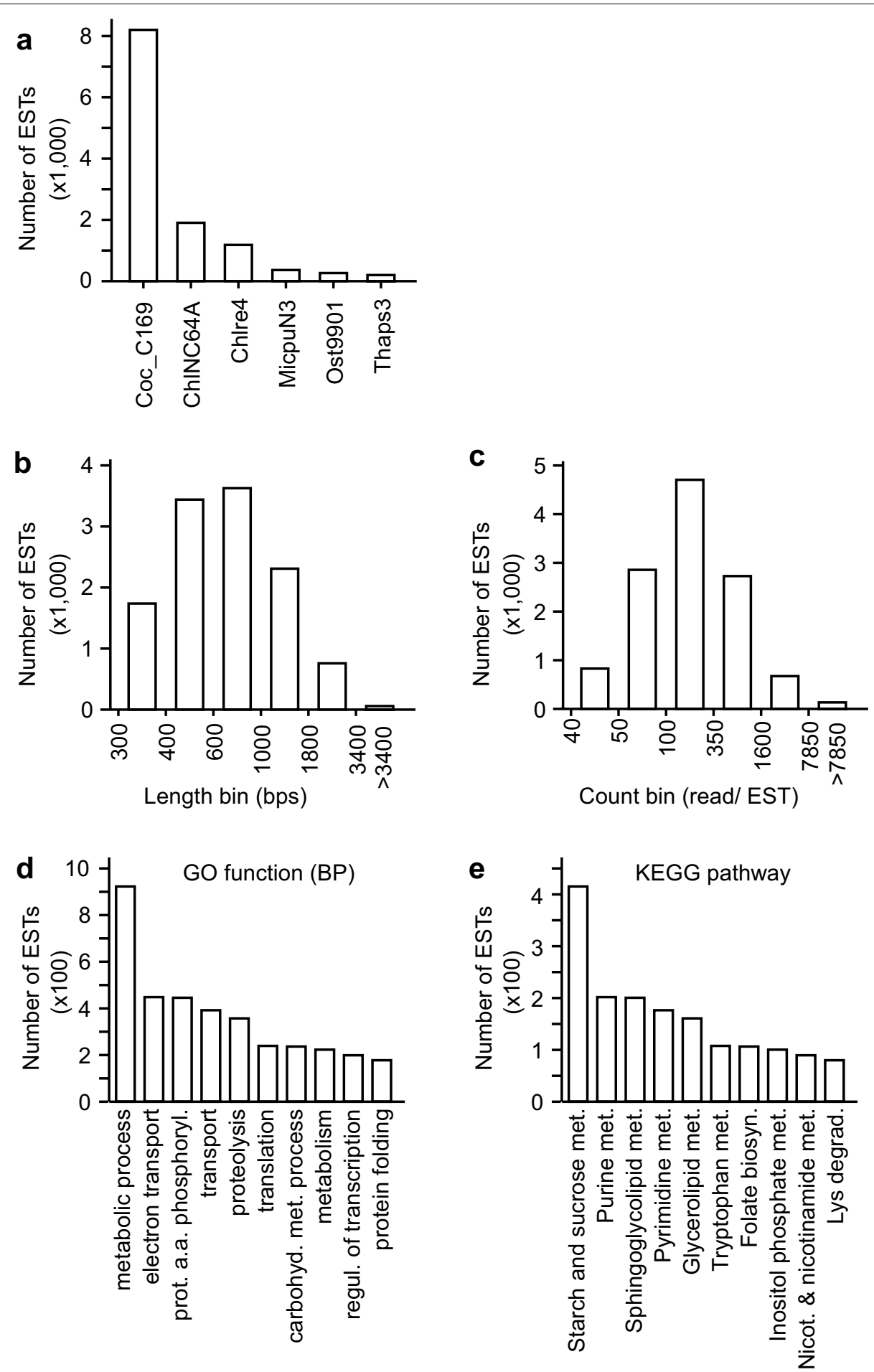

Fig. 3 Characteristics of the de novo-assembled B. braunii 779 transcriptome. a Distribution of number of best-hits derived from the genome of various algal species. X- and Y-axis indicate the genome of algal species used and the number of ESTs in B. braunii 779. $\mathbf{b}$ Length and $\mathbf{c}$ count distribution of the 21,292 annotated ESTs in B. braunii 779. X-and Y-axis indicate the EST length (left panel) and count (right panel) and number of ESTs, respectively. d Ten GO biological process categories associated with the most number of ESTs in the transcriptome of B. braunii 799. e Ten KEGG pathways associated with the most number of ESTs in the transcriptome of B. braunii 799. 


\section{a}

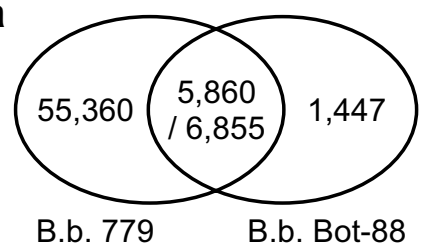

$(61,220)$

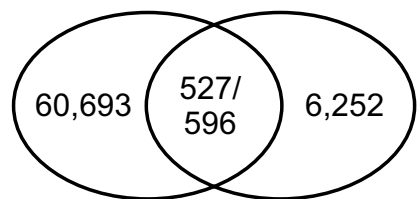

B.b. 779

$(61,220)$

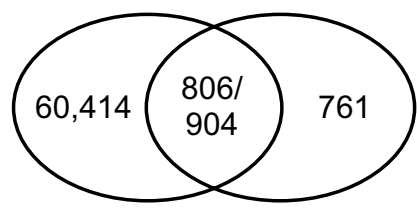

B.b. 779

$(61,220)$

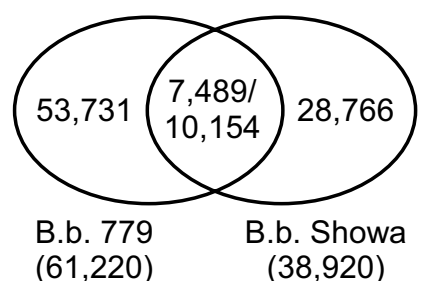

$(61,220)$

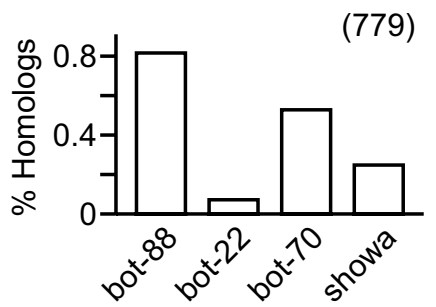

b
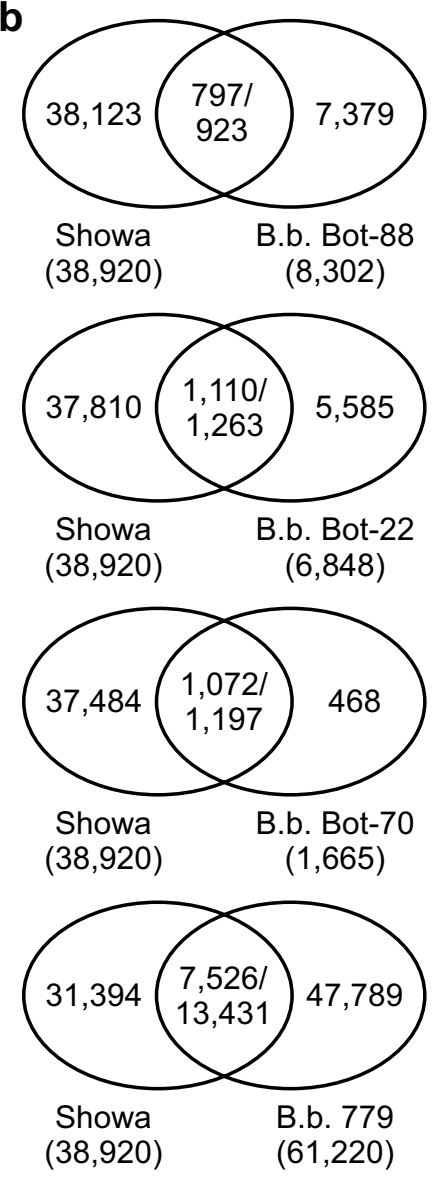

(showa)

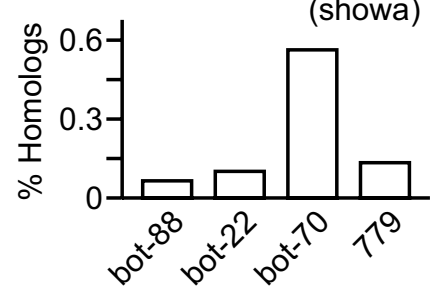

C
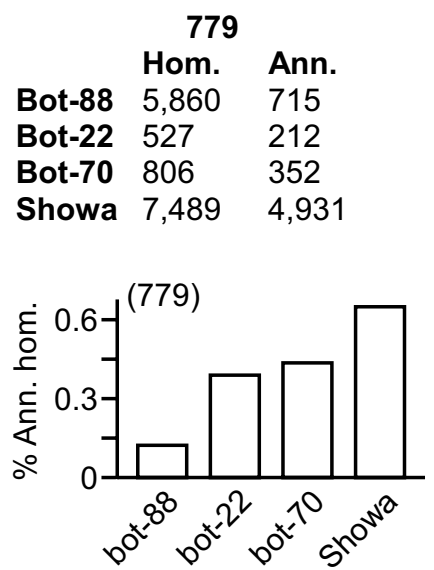

Fig. 4 Highest level of similarities is found between transcriptomes of the same race $B$. braunii strains. a Highest level of similarity is observed between transcriptomes of B. braunii Bot-88 and 779. Venn diagrams show the homologous ESTs between the query transcriptome (e.g., Bot-88, Bot-22, Bot-70, or Showa) and the subject transcriptome 779. Percent of homologous ESTs in query transcriptomes is shown at the bottom. $\mathbf{b}$ Highest level of similarity is found between the query transcriptomes of B. braunii Showa and Bot-70. Percent of homologous ESTs in query transcriptomes is shown at the bottom. c Lowest percentage of the known sequences is found in the homologous ESTs between B. braunii 779 and Bot-88. Number of all homologous (Hom.) ESTs and annotated (Ann.) ESTs between B. braunii 779 and other transcriptomes are listed on the upper panel. A bar plot at bottom shows the percent of annotated ESTs in various sets of homologous ESTs in upper panel.

component-encoding ESTs were also enriched in window 1 (i.e., fold change $>2$, $\mathrm{p}$ value $<0.05$ after Bonferroni correction). On the other hand, ESTs associated with the TCA cycle and pentose phosphate pathway were enriched the window containing the least abundant ESTs or in window 23 (i.e., fold change $>2$, p value $<0.05$ after Bonferroni correction). Photosynthesis (or light harvesting) and carbon fixation were essential for cell growth under photoautotrophic conditions. High-level expression of these functions in log-phase B. braunii cells indicated that the slow growth was not a result of inefficient energy metabolisms, rather than its devoting major portion of its energy flow to the lipid and hydrocarbon synthesis. 
Table 3 List of the top 30 most abundant ESTs in log-phase B. braunii 779 cells

\begin{tabular}{|c|c|c|c|c|}
\hline $\mathbf{R k}^{\mathrm{a}}$ & EST_ID ${ }^{\mathrm{b}}$ & BH_ID ${ }^{c}$ & FPKM $^{d}$ & Description $^{\mathrm{e}}$ \\
\hline 1 & C72167_g1_i4 & ChINC64A_1|59790 & $12,471.445$ & Photosynthesis, light harvesting \\
\hline 2 & C72167_g1_i8 & Coc_C169_1|58975 & $10,031.55$ & Photosynthesis, light harvesting \\
\hline 3 & C72102_g4_i5 & Coc_C169_1|27246 & $7,119.695$ & Photosynthesis, light harvesting \\
\hline 4 & C72167_g1_i2 & Coc_C169_1|58975 & $6,831.27$ & Photosynthesis, light harvesting \\
\hline 5 & C72167_g1_i6 & Coc_C169_1|27246 & $6,731.515$ & Photosynthesis, light harvesting \\
\hline 6 & C72102_g4_i4 & Coc_C169_1|58975 & $4,540.985$ & Photosynthesis, light harvesting \\
\hline 7 & C72167_g1_i7 & Coc_C169_1|27246 & $3,366.845$ & Photosynthesis, light harvesting \\
\hline 8 & C72102_g4_i1 & Coc_C169_1/58975 & $2,639.815$ & Photosynthesis, light harvesting \\
\hline 9 & c68174_g2_i1 & Coc_C169_1|30477 & $2,360.575$ & $60 S$ ribosomal protein $\mathrm{L} 23$ \\
\hline 10 & c68658_g2_i1 & Coc_C169_1|35121 & $2,110.295$ & Photosynthesis, light harvesting \\
\hline 11 & c57826_g1_i1 & Coc_C169_1|44136 & $1,790.37$ & Photosynthesis, light harvesting \\
\hline 12 & c58876_g1_i1 & Coc_C169_1|28488 & $1,713.8$ & Photosynthesis, light harvesting \\
\hline 13 & c54674_g1_i1 & Coc_C169_1/64185 & $1,710.555$ & Photosynthesis, light harvesting \\
\hline 14 & C72326_g1_i2 & Coc_C169_1/67011 & $1,573.705$ & Photosynthesis, light harvesting \\
\hline 15 & C72522_g1_i1 & Coc_C169_1|34109 & $1,538.055$ & Fructose-biphosphate aldolase \\
\hline 16 & c70416_g1_i1 & Coc_C169_1|27513 & $1,432.875$ & Translation elongation factor EF-1 \\
\hline 17 & c65010_g1_i1 & Coc_C169_1|19247 & $1,314.1$ & Photosynthesis, light harvesting \\
\hline 18 & c62135_g1_i1 & Chlre4|184775 & $1,184.59$ & Photosynthesis, light harvesting \\
\hline 19 & C72592_g1_i1 & Coc_C169_1|35576 & $1,170.825$ & Enolase \\
\hline 20 & C71666_g1_i1 & Chlre4|140045 & $1,082.22$ & Ubiquitin and ubiquitin-like proteins \\
\hline 21 & c58350_g1_i1 & Coc_C169_1|28397 & $1,073.435$ & $60 S$ ribosomal protein $L 35$ \\
\hline 22 & C72102_g5_i1 & Coc_C169_1|28488 & 977.63 & Photosynthesis, light harvesting \\
\hline 23 & C70207_g1_i2 & ChINC64A_1|59626 & 859.85 & Photosynthesis, light harvesting \\
\hline 24 & C70122_g2_i2 & Coc_C169_1|53355 & 839.985 & Glyceraldehyde 3-phosphate dehydrogenase \\
\hline 25 & c69841_g1_i1 & Coc_C169_1|25284 & 823.07 & Photosynthesis, light harvesting \\
\hline 26 & c69841_g1_i3 & Coc_C169_1|25284 & 818.15 & Photosynthesis, light harvesting \\
\hline 27 & C57090_g1_i1 & Coc_C169_1|26429 & 790.785 & $60 S$ ribosomal protein $L 44$ \\
\hline 28 & c68918_g1_i1 & Coc_C169_1|37969 & 772.455 & Photosynthesis, light harvesting \\
\hline 29 & c61023_g1_i1 & Coc_C169_1|34169 & 689.075 & $60 S$ ribosomal protein $\mathrm{L} 30$ \\
\hline 30 & c68918_g1_i2 & Coc_C169_1|37969 & 676.53 & Photosynthesis, light harvesting \\
\hline
\end{tabular}

${ }^{a}$ Rk stands for rank based on average level of FPKM.

b EST_ID for ID of the B. braunii 779 ESTs.

c BH_ID for EST's best-hit consistent of species and protein ID.

d FPKM for average level of individual ESTs.

e Description is based on $\mathrm{GO}$ annotations.

ESTs encoding photosynthesis and ribosome functions are down-regulated upon ND in B. braunii 779

To investigate changes of transcriptional activities upon ND, levels of individual ESTs after ND were compared with those prior to ND. We assumed that if a subset of ESTs associated with a pathway was enriched within a window of 1024 ESTs ranked by ratio (i.e., fold change $>2$, $\mathrm{p}$ value $<0.05$ after Bonferroni correction), the subset of ESTs was potentially co-regulated by a common transcription regulatory network in response to ND. Using the sliding window approach along the sorted ESTs based on rank by ratio, we found that subsets of ESTs associated with $3 \mathrm{GO}$ biological processes (i.e., carbohydrate metabolism, glycolysis, and photosynthesis) and 6 KEGG metabolic pathways (i.e., tyrosine metabolism, fluorene degradation, TCA cycle, glycolysis/gluconeogenesis, pentose phosphate pathway, carbon fixation) plus ribosome subunit were enriched in one of the 23 sliding windows (Fig. 6a).

We found that subsets of ESTs associated with carbohydrate metabolism and glycolysis processes or TCA cycle, glycolysis/gluconeogenesis, pentose phosphate pathway, and carbon fixation metabolic pathways were enriched in the top most up-regulated window (see Fig. 6a). This was puzzling because cell growth would be arrested upon ND. However, it was proposed that ND induced oxidative 


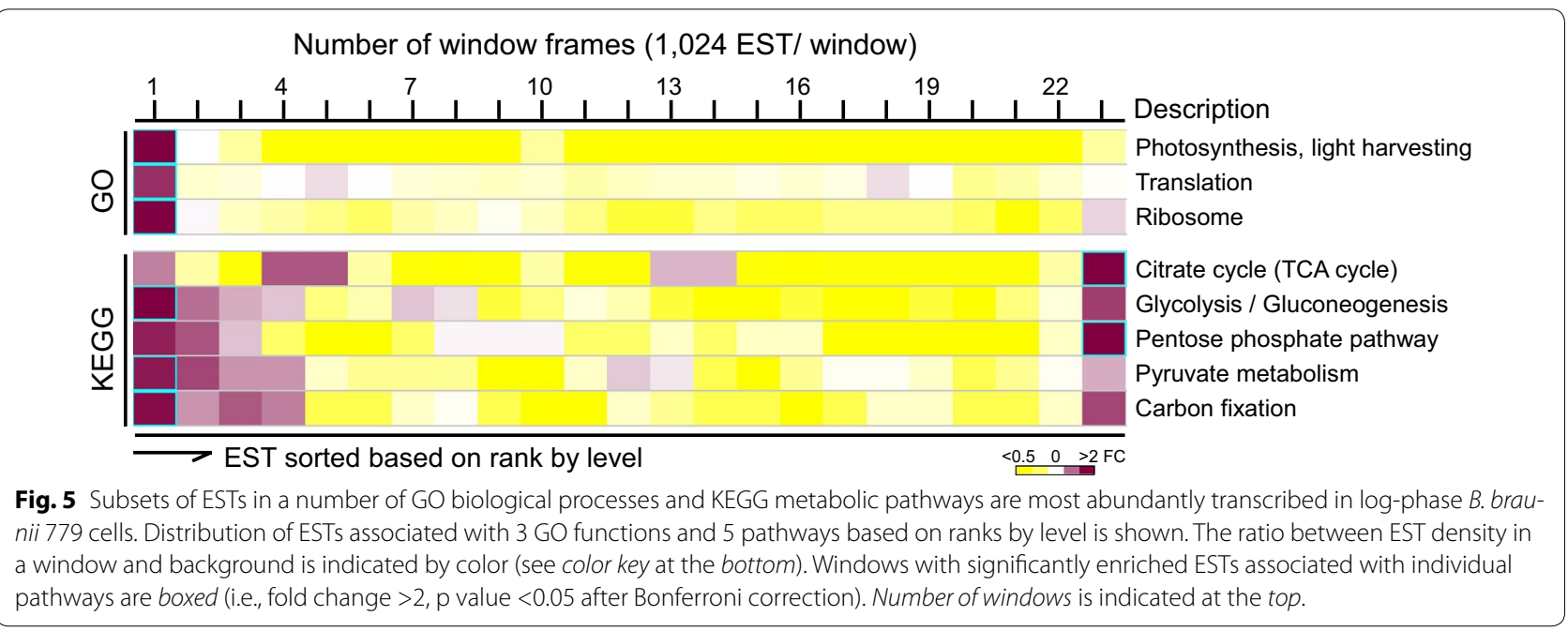

stress that in turn triggered reconfiguration of metabolic flux to provide the reducing power for the main cellular redox systems [42, 43]. Hence, we hypothesized that the up-regulation of citrate cycle, glycolysis/gluconeogenesis, pentose phosphate pathway, and carbon fixation metabolisms was a result of reconfiguration of carbohydrate metabolisms for production of reducing power upon ND.

To test this possibility, we investigated all ESTs that were associated with unspecific dehydrogenase activity based on annotation of "dehydrogenase". We found that unspecific dehydrogenase ESTs were enriched in 20 metabolisms (i.e., fold change $>2$, p value $<0.05$ ) including citrate cycle, glycolysis/gluconeogenesis, pentose phosphate pathway, and carbon fixation metabolisms. ESTs associated with unspecific dehydrogenase were found to be up-regulated upon ND (i.e., fold change $>1.5$, $\mathrm{p}$ value $<0.05$ after Bonferroni correction) (Fig. 6b). This result implied that up-regulation of the unspecific dehydrogenase-enriched metabolisms such as citrate cycle, glycolysis/gluconeogenesis, pentose phosphate pathway, and carbon fixation is likely a result of reconfiguration of metabolic flux to provide the reducing power for the main cellular redox systems.

We found that ESTs encoding photosynthesis (or light harvesting) and ribosome functions were enriched in the most down-regulated windows (i.e., ranked at bottom by ratio) (see Fig. 6a). It was proposed that excess proteins involved in light harvesting and protein synthesis were used as nitrogen-rich molecules to recycle nitrogen during ND [22]. To test this possibility, we measured chlorophyll content and photosynthetic yield in cells prior to and after ND. Although transcription level of ESTs associated with light harvesting and ribosome function was dramatically down-regulated, the level of chlorophyll and photosynthetic yield was only marginally affected
(Fig. 6c, d). This result implied that excess transcripts encoding photosynthesis function and ribosomes were recycled for nitrogen hardly affecting the photosynthetic efficiency upon ND in B. braunii 779.

Up-and down-regulated ESTs induced by ND appear to be low- and high-abundant ESTs in log-phase B. braunii 779 cells, respectively

Based on the duplicate, read count per EST was estimated by RSEM [44] and subsequently the differential expression level was determined using EdgeR [45]. To this end, we obtained 593 and 116 differentially up- and down-regulated ESTs in response to ND in B. braunii 779 , respectively, based on a cutoff of fold change $>2$ and $\mathrm{p}$ value $<0.05$ (see "Methods", for a complete list of differentially transcribed ESTs, see Additional file 7: Table S12). Lists of the top 25 up-regulated and down-regulated ESTs are shown in Tables 4 and 5 . We found that many up-regulated ESTs upon ND (i.e., fold change $>2$, p value $<0.05$ ) appeared to be the least abundant ESTs prior to ND (Fig. 7). Though nitrogen metabolic pathway was not up-regulated upon ND, we found that 7 ESTs (i.e., 1 ammonia permease, 2 glutamine synthases, and 4 glutamate synthases) out of the top 25 most up-regulated ones were involved in nitrogen metabolism (see Table 4). On the other hand, many down-regulated ESTs upon ND tended to be relatively highly abundant in cells prior to ND (see Fig. 7).

\section{Transcriptional alteration of ESTs involved in alkadiene/ triene-related biosynthesis of VLCFA upon ND in B. braunii 779}

A subset of 55 enzymes that were potentially involved in the biosynthesis of VLCFA was proposed by Baba et al., out of which they detected 19 enzymes in the A race $B$. 


\section{a}
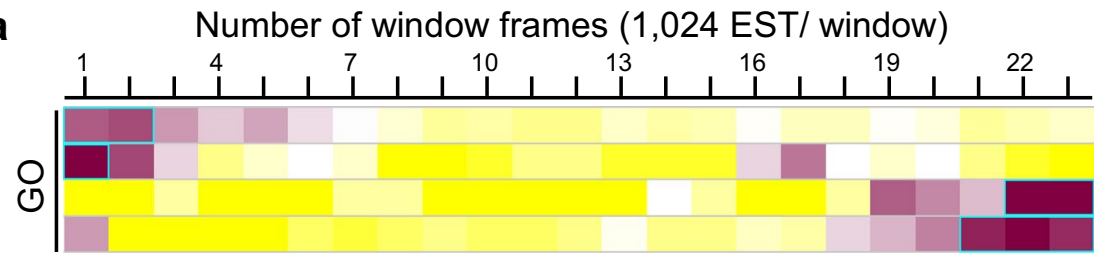

Description

Carbohydrate metabolic process

Glycolysis

Photosynthesis, light harvesting

Ribosome

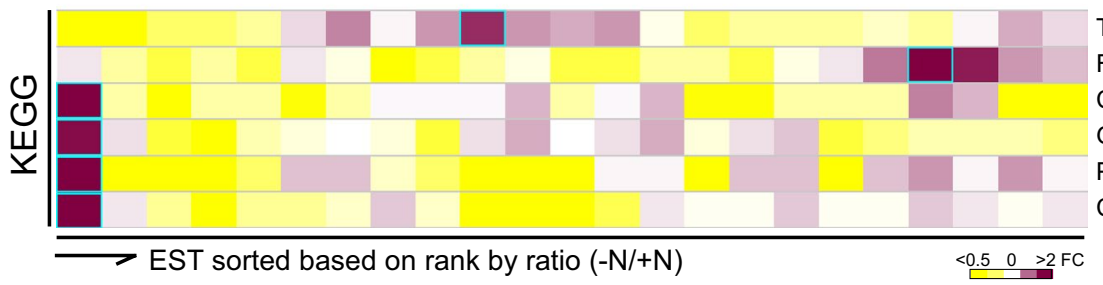

Tyrosine metabolism

Fluorene degradation

Citrate cycle (TCA cycle)

Glycolysis / Gluconeogenesis

Pentose phosphate pathway

Carbon fixation

Up-regulation $\longrightarrow$ Down-regulation

b

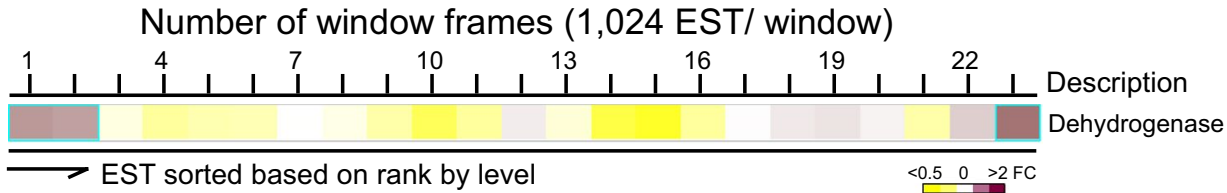

Number of window frames (1,024 EST/ window)
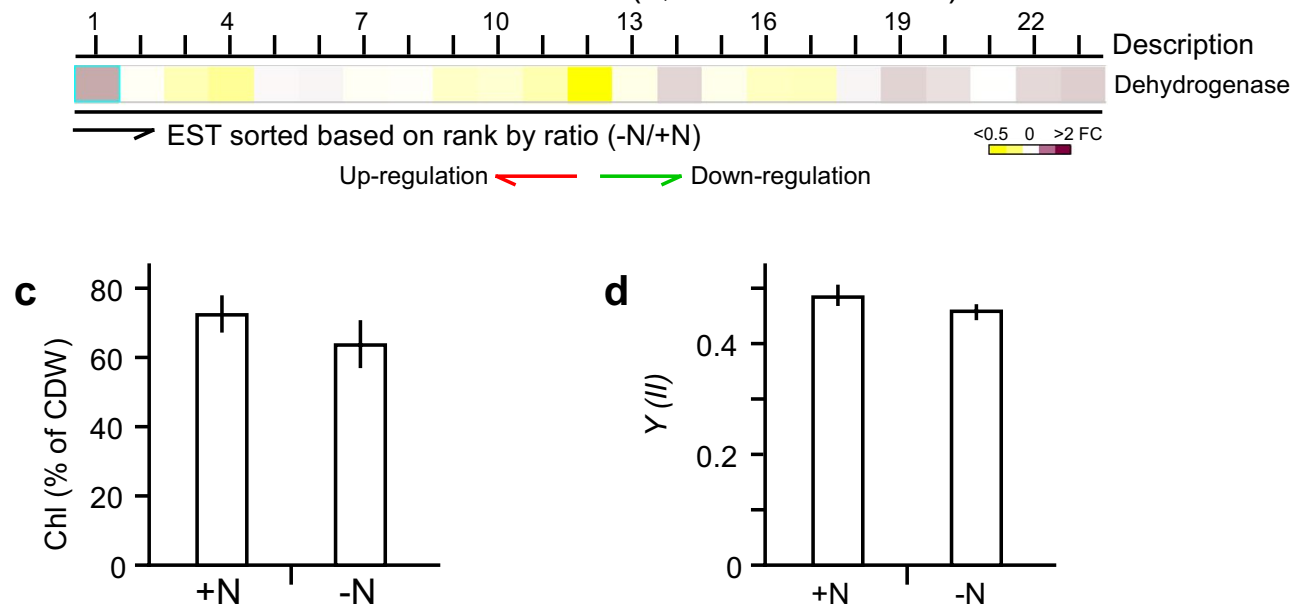

Fig. 6 Subsets of ESTs in a number of processes and pathways are most up-regulated and down-regulated upon ND in B. braunii 779 cells. a Distribution of ESTs associated with 4 GO functions and 6 KEGG pathways based on ranks by ratio. b Distribution of ESTs associated with unspecific dehydrogenase based on ranks by level (upper panel) and ratio (lower panel). The display is identical to Fig. 5. c Levels of chlorophyll content. The bar plot shows the average level of chlorophyll contents in cells prior to $(+\mathrm{N})$ and after $(-\mathrm{N}) \mathrm{ND}$. Error bar is derived from triplicates. $\mathbf{d}$ Efficiency of quantum yield of photosystem II. The bar plot shows the average level of efficiency of quantum yield ( $Y$ ) of photosystem II in cells prior (+N) to and after $(-\mathrm{N}) \mathrm{ND}$.

braunii Bot-88 [24]. Based on association with these EC numbers, we detected 20 enzymes in the B. braunii 779 transcriptome, of which, 11 were those previously found in B. braunii Bot-88 [24], suggesting that A race B. braunii strains share many VLCFA biosynthetic enzymes (i.e., $\mathrm{p}$ value $=0.0137)$.
We found that each enzyme was encoded by various copies of the non-redundant ESTs (Fig. 8a). Levels of individual copies of non-redundant ESTs ranged from 1.9 to 110 FPKM in cells prior to and from 1.9 to 80 FPKM after ND based on the average of two repeats. There were 1 significantly up-regulated and 3 down-regulated ESTs 
Table 4 List of the top 25 most up-regulated ESTs upon ND in B. braunii 779

\begin{tabular}{|c|c|c|c|c|c|}
\hline $\mathbf{R k}^{\mathrm{a}}$ & EST_ID ${ }^{b}$ & BH_ID ${ }^{c}$ & $\log F C$ & $p$ value & Description $^{d}$ \\
\hline 1 & C39922_g1_i1 & Ost9901_3|32601 & 12.06 & $1.2 \mathrm{E}-13$ & Ammonia permease \\
\hline 2 & c63389_g1_i3 & Coc_C169_1|23517 & 10.56 & 4.1E-09 & Glutamine synthetase \\
\hline 3 & c9966_g1_i1 & Chlre4|188119 & 10.36 & $3.6 \mathrm{E}-09$ & Oxidoreductase \\
\hline 4 & c27174_g1_i1 & Coc_C169_1|52379 & 9.57 & 8.9E-09 & Glycogen phosphorylase \\
\hline 5 & c60040_g1_i1 & Coc_C169_1|32937 & 9.49 & $1.3 \mathrm{E}-08$ & Pyruvate kinase \\
\hline 6 & C90800_g1_i1 & ChINC64A_1|143431 & 9.48 & $2.3 \mathrm{E}-09$ & Glutamine synthetase \\
\hline 7 & C71872_g1_i2 & Ost9901_3|50765 & 9.19 & $2.8 \mathrm{E}-10$ & Sulfite reductase \\
\hline 8 & c52649_g1_i1 & Coc_C169_1|52379 & 8.65 & $1.0 \mathrm{E}-07$ & Glycogen phosphorylase \\
\hline 9 & c81537_g1_i1 & Chlre4|206116 & 8.04 & 4.1E-08 & Unknown \\
\hline 10 & c67134_g2_i2 & MicpuN3|63658 & 7.94 & $1.3 \mathrm{E}-06$ & Glutamate synthase \\
\hline 11 & C78877_g1_i1 & Chlre4|140452 & 7.77 & $5.1 \mathrm{E}-07$ & Unknown \\
\hline 12 & c49437_g1_i2 & MicpuN3|97997 & 7.67 & $1.2 \mathrm{E}-06$ & Unknown \\
\hline 13 & c9210_g1_i2 & Chlre4|132210 & 7.66 & $1.7 \mathrm{E}-07$ & 3-phosphoglycerate kinase \\
\hline 14 & c70762_g1_i7 & Chlre4|140487 & 7.65 & $1.8 \mathrm{E}-07$ & Glutamate synthase \\
\hline 15 & c59003_g1_i1 & MicpuN3|63658 & 7.45 & $9.2 \mathrm{E}-06$ & Glutamate synthase \\
\hline 16 & C19522_g1_i1 & Thaps3|21748 & 7.24 & $6.4 \mathrm{E}-07$ & Fructose 1,6-bisphosphate aldolase \\
\hline 17 & c20385_g1_i1 & Ost9901_3|30705 & 7.14 & $9.0 \mathrm{E}-07$ & Cohesin subunit \\
\hline 18 & c27189_g1_i1 & Thaps3|27187 & 6.88 & $2.4 \mathrm{E}-07$ & Transaldolase \\
\hline 19 & c24873_g1_i2 & MicpuN3|89262 & 6.88 & $1.3 \mathrm{E}-06$ & FOG: RCC1 domain \\
\hline 20 & c51175_g1_i1 & Coc_C169_1|29458 & 6.87 & $3.8 \mathrm{E}-06$ & Citrate synthase \\
\hline 21 & c47749_g1_i1 & Coc_C169_1|9065 & 6.87 & $1.0 \mathrm{E}-07$ & Transcription factors \\
\hline 22 & c61180_g1_i2 & Chlre4|205746 & 6.79 & $7.9 \mathrm{E}-06$ & Glutamate synthase \\
\hline 23 & c2096_g1_i1 & MicpuN3|82943 & 6.76 & $4.0 \mathrm{E}-07$ & RNA Helicase \\
\hline 24 & c36160_g1_i3 & Thaps3|262283 & 6.74 & $5.6 \mathrm{E}-07$ & Acyl-CoA synthetase \\
\hline 25 & c27223_g1_i2 & Thaps3|269844 & 6.71 & $4.0 \mathrm{E}-07$ & UDP-glucuronosyl transferase \\
\hline
\end{tabular}

a $R$ k stands for rank (i.e., by ratio).

b EST_ID for B. braunii ESTs.

c BH_ID for ID of the best-hit.

${ }^{d}$ Description is based on the KOG annotation associated with the 6 completed algal genomes in JGI (see text).

(i.e., fold change $>2$, p value $<0.05$ ). After summation of levels of all ESTs associated with the same enzyme, we found that the summative level of all enzymes tested was not greatly altered (i.e., fold change $<2$ ) upon ND except for acetyl-CoA oxidase (i.e., >twofold decrease) (Fig. 8b, for summative level of individual enzymes, see Additional file 8: Table S13). This result indicated that no apparent transcriptional alteration for most enzymes (i.e., not individual ESTs) involved in VLCFA biosynthesis upon ND in B. braunii 779.

\section{Transcriptional alteration of ESTs involved} in botryococcene or squalene-related biosynthesis upon ND in B. braunii 779

Transcriptome of the $B$ race $B$. braunii Showa was well assembled and comprehensively annotated [21]. To examine whether the transcription of enzymes involved in biosynthesis of botryococcene or squalene was altered upon ND in A race B. braunii 779, we compared the ESTs between B. braunii 779 and Showa based on the association with EC numbers. To this end, we found 58 and 42 enzymes related to botryococcene or squalene biosynthesis were associated with curated and machine-assembled ESTs in B. braunii Showa [21], respectively (see "Methods"). Of the 58 enzymes associated with curated ESTs in B. braunii Showa, 13 (or 22.4 \%) were found to associate with a total of 29 ESTs in B. braunii 779 (Fig. 9a, left panel). Based on the summation of all ESTs associated with the same enzyme, we found that summative transcription level of all enzymes was not dramatically altered upon ND (i.e., fold change $<2$ ), except for TKTL and G44OX (i.e., >twofold increase) (Fig. 9a, right panel, for summative level of individual enzymes, see Additional file 9: Table S14). On the other hand, 23 out of 42 (or $54.8 \%$ ) machine-assembled enzymes in B. braunii Showa were found to associate with 171 ESTs in $B$. braunii 779, five of which associated with 19 or more ESTs (Fig. 9b, left panel). Similar to that of curated enzymes, 
Table 5 List of the top 25 most down-regulated ESTs upon ND in B. braunii 779

\begin{tabular}{|c|c|c|c|c|c|}
\hline $\mathrm{Rk}^{\mathrm{a}}$ & EST_ID ${ }^{b}$ & BH_ID ${ }^{c}$ & $\log \mathrm{FC}$ & $p$ value & Description $^{d}$ \\
\hline 1 & c73365_g3_i6 & Coc_C169_1|45719 & -4.55 & $6.4 \mathrm{E}-03$ & Unknown \\
\hline 2 & C54903_g1_i1 & Coc_C169_1|45718 & -4.40 & $2.5 \mathrm{E}-03$ & Tyrosine kinase \\
\hline 3 & C72890_g1_i2 & ChINC64A_1|144962 & -4.20 & $1.8 \mathrm{E}-03$ & Prolylcarboxypeptidase \\
\hline 4 & c69499_g1_i5 & Chlre4|149722 & -4.18 & $4.5 \mathrm{E}-03$ & Unknown \\
\hline 5 & c66708_g1_i4 & Coc_C169_1|65911 & -3.98 & $2.8 \mathrm{E}-04$ & Collagens \\
\hline 6 & C72757_g1_i1 & Coc_C169_1|17756 & -3.54 & $1.5 \mathrm{E}-03$ & Isocitrate lyase \\
\hline 7 & c66635_g1_i1 & ChINC64A_1|139124 & -3.46 & $2.8 \mathrm{E}-03$ & Nuclear receptor coregulator \\
\hline 8 & c32919_g1_i2 & Coc_C169_1|18195 & -3.44 & $2.1 \mathrm{E}-04$ & Aspartate aminotransferase \\
\hline 9 & C71206_g1_i2 & Coc_C169_1/62318 & -3.36 & $2.0 \mathrm{E}-02$ & UDP-glucuronosyl transferase \\
\hline 10 & c68672_g1_i4 & Coc_C169_1|65911 & -3.31 & $9.4 \mathrm{E}-04$ & Collagens \\
\hline 11 & C55715_g1_i1 & Coc_C169_1|68180 & -3.27 & $6.9 \mathrm{E}-04$ & $\mathrm{Ca}^{2+}$-dependent protein kinase \\
\hline 12 & C71767_g1_i2 & Chlre4|104719 & -3.23 & $5.1 \mathrm{E}-03$ & ATP-dependent RNA helicase \\
\hline 13 & c69499_g1_i4 & Chlre4|149722 & -3.16 & 1.7E-02 & Unknown \\
\hline 14 & C73427_g1_i1 & Coc_C169_1|35165 & -3.01 & $1.0 \mathrm{E}-02$ & O-linked GlcNAc transferase \\
\hline 15 & c66185_g1_i1 & Coc_C169_1|33465 & -3.01 & $1.6 \mathrm{E}-02$ & Unknown \\
\hline 16 & c67100_g1_i1 & Chlre4|130199 & -3.01 & $2.2 \mathrm{E}-02$ & Acetylglutamate kinase \\
\hline 17 & C71718_g1_i18 & Coc_C169_1|68054 & -3.01 & 4.4E-03 & $\mathrm{Ca}^{2+}$-permeable cation channel \\
\hline 18 & c70935_g1_i8 & Coc_C169_1|13500 & -3.00 & 4.4E-03 & Unknown \\
\hline 19 & c51991_g1_i1 & Coc_C169_1|63373 & -2.99 & $1.2 \mathrm{E}-02$ & Diacylglycerol acyltransferase \\
\hline 20 & c74265_g5_i3 & Coc_C169_1|30369 & -2.94 & $3.9 \mathrm{E}-03$ & Malate synthase \\
\hline 21 & C73301_g5_i1 & Coc_C169_1|17144 & -2.94 & $1.5 \mathrm{E}-02$ & Serine/threonine protein kinase \\
\hline 22 & C71077_g1_i3 & ChINC64A_1|59702 & -2.94 & $8.1 \mathrm{E}-03$ & Unknown \\
\hline 23 & C73505_g1_i1 & Coc_C169_1|41290 & -2.92 & $2.0 \mathrm{E}-03$ & Unknown \\
\hline 24 & C74003_g1_i7 & Coc_C169_1|35165 & -2.89 & $6.3 \mathrm{E}-03$ & O-linked GlcNAc transferase \\
\hline 25 & c62188_g1_i1 & Coc_C169_1|11065 & -2.89 & $9.6 \mathrm{E}-03$ & Sensory transduction histidine kinase \\
\hline
\end{tabular}

${ }^{a}$ Rk stands for rank (i.e., by inverse ratio).

${ }^{\mathrm{b}}$ EST_ID for B. braunii ESTs.

c BH_ID for ID of the best-hit.

${ }^{d}$ Description is based on the KOG annotation associated with the 6 completed algal genomes in JGI (see text).

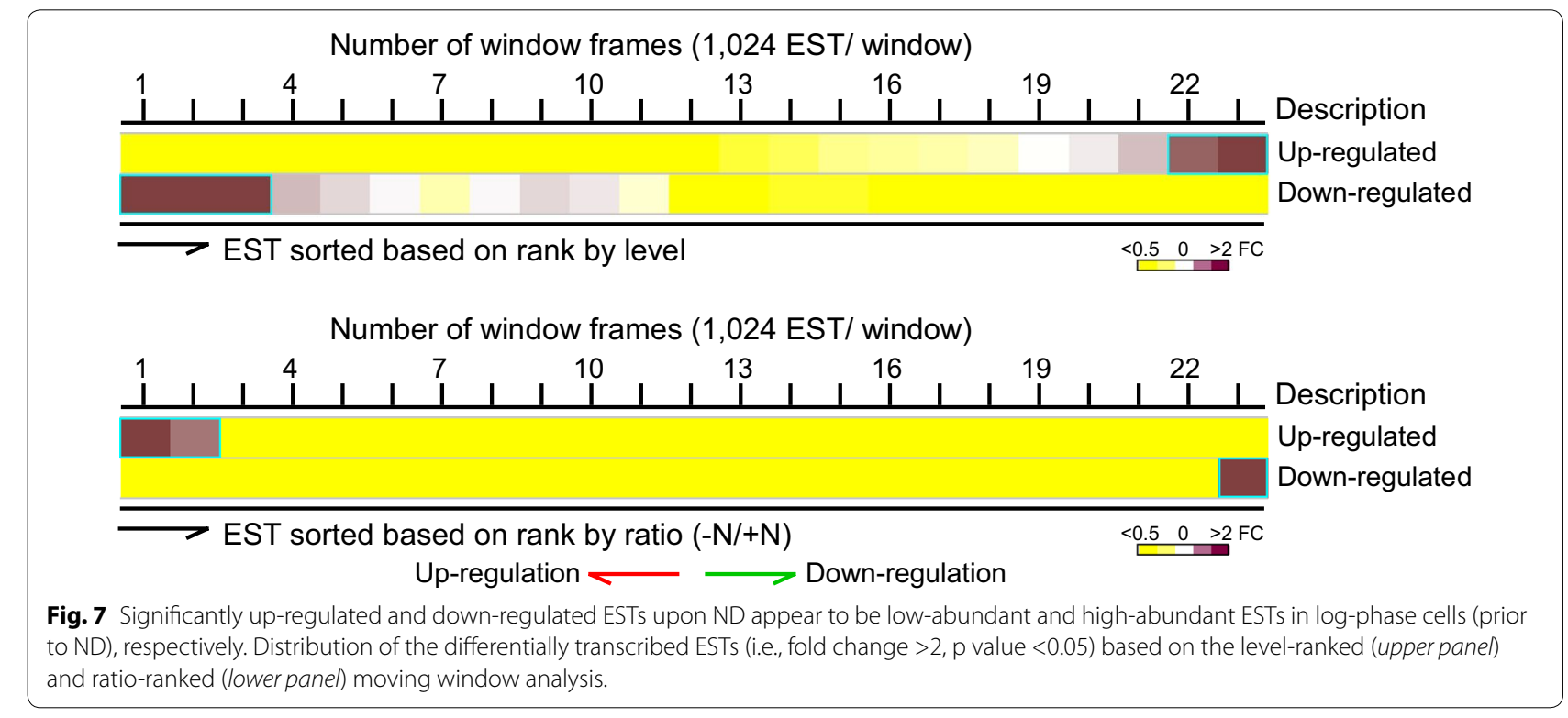




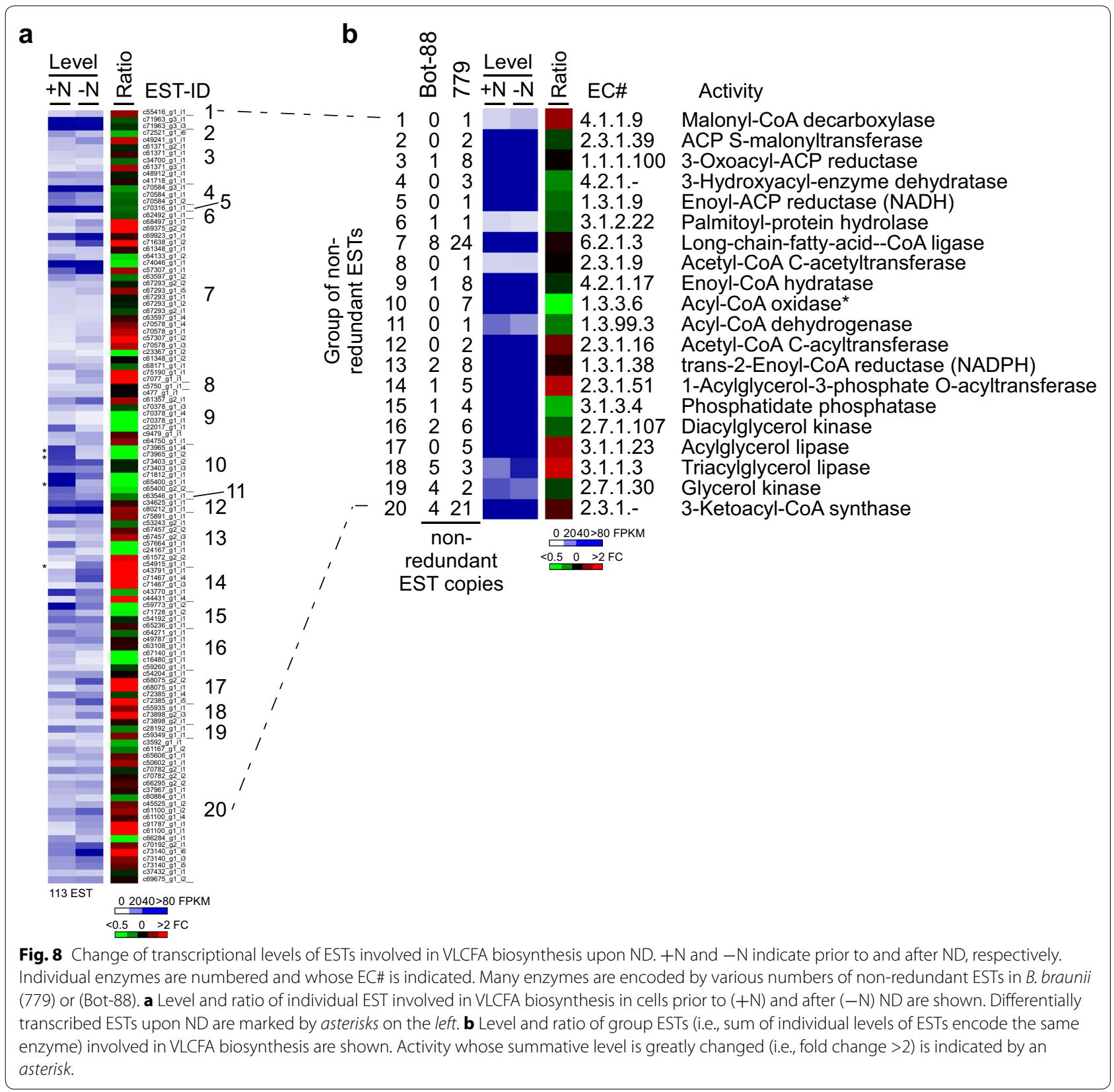

transcriptional level of all enzymes was not dramatically altered upon ND (i.e., fold change $<2$ ), except for DGAT and HK (i.e., >twofold increase), based on the summative level of all ESTs associated with the same enzyme (Fig. 9b, right panel, for summative level of individual enzymes, see Additional file 10: Table S15). These results suggested that transcriptional level of most enzymes involved in botryococcene or squalene biosynthetic pathways was not significantly altered upon ND in A race $B$. braunii 779.

\section{Discussion}

The colonial green microalga $B$. braunii is unique for its accumulation of hydrocarbons, which is applicable for biofuel production. However, slow growth has impeded its application for large-scale production of hydrocarbons. Unlike our previous report that a rapidly growing subisolate from a culture of $B$. braunii UTEX572 has turned out to be a Botryosphaerella (UTEX2629) containing no hydrocarbons [22], we show, in this study, that the moderately growing subisolate from a culture 


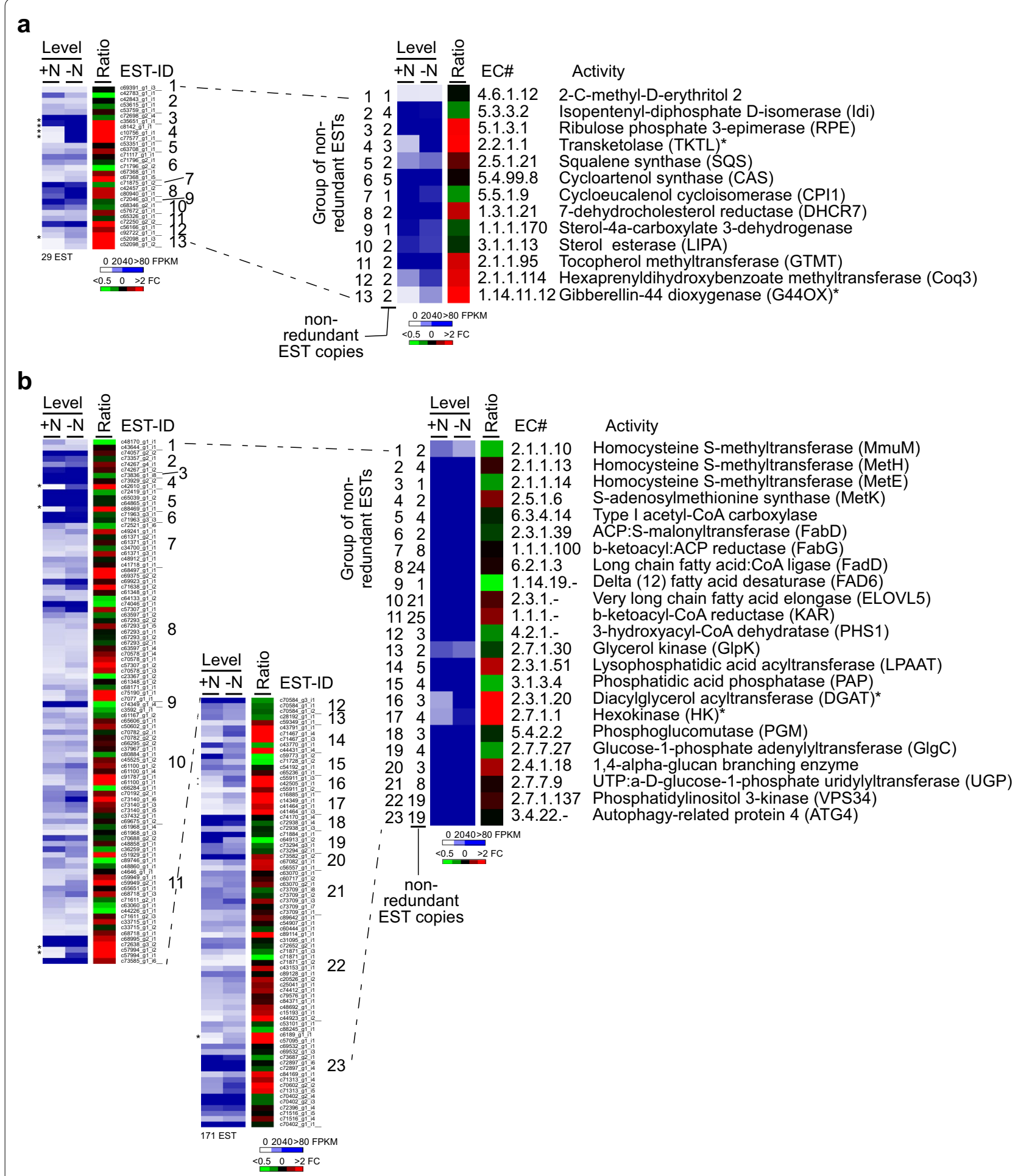

Fig. 9 Change of transcriptional levels of ESTs involved in botryococcene or squalene biosynthesis upon ND. The display is identical to Fig. 8. a ESTs homologous to curated and machine-assembled $\mathbf{b}$ enzymes involved in botryococcene or squalene biosynthesis in B. braunii Showa [21]. The display is identical to Fig. 8. 
of Botryococcus sp. CCALA-779 belongs to the A race B. braunii based on the results of $18 \mathrm{~S}$ rRNA sequencebased phylogenetic analysis and GC-MS analysis of hydrocarbon compositions (see Fig. 2). The lipid content of this moderately growing $B$. braunii 779 strain under phototrophic conditions can be greatly enhanced by ND, making it attractive for potential production of hydrocarbons at large scale.

In comparison with that of model green algal organisms Chlorella variabilis [35] and Chlamydomonas reinhardtii [36], growth rate of $B$. braunii is very slow (i.e., population doubling time of less than a day versus 5-7 days). In this study, we show that, according to the pathway-based transcriptomic analysis, energy metabolic ESTs are highly abundant in the log-phase cells, indicating that the slow growth rate of $B$. braunii species is not a result of inefficient energy metabolic activity in cells. It is likely that energy flow generated from photosynthesis in B. braunii cells is largely directed to the biosynthesis of lipid and hydrocarbon. We propose that if the direction of energy flow could be controlled in cells, the productivity would be improved by first directing the energy flow to population growth. After reaching the maximum density of cell population, energy flow would be re-directed to lipid and hydrocarbon synthesis.

It has been shown that the modes of hydrocarbon biosynthesis in A race B. braunii Bot- 88 and $\mathrm{B}$ race $B$. braunii Showa, Bot-22, and Bot-70 are very different according to the 454 sequencing-based transcriptomic analyses [21, 23-25]. In this study, we performed transcriptomic analysis of the B. braunii 779 using the Illumina sequencing-based platform. Comparative analysis indicates that highest percentage of sequence similarities is found between transcriptomes of the same race such as $A$ race $B$. braunii strains 779 and Bot- 88 and $B$ race strains Showa and Bot-70 (see Fig. 4). However, we find that transcriptome of Bot-22 shares little similarity to either B race B. braunii Showa or A race 779 strains, suggesting that it represents no $A$ or $B$ race transcriptome. The difference between transcriptomes of $A$ and $B$ races implies that they belong to different sub-species.

It is worth noting that $40-60 \%$ of the homologous ESTs between B. braunii 779 and other B race strains are known sequences (or having best-hit in the 6 algal genomes used in this study). On the other hand, only $12 \%$ of the homologous ESTs between A race B. braunii 779 and Bot-88 are known sequences. Majority of the homologous ESTs in A race transcriptomes are unknown sequences, suggesting that there are many novel genes in $B$. braunii. Given that many enzymes encoding VLCFA biosynthesis have not been found in A race $B$. brau$n i i$, it is possible that sequences encoding these enzymatic activities may not be currently known. Hence, we propose that $B$. braunii provides a rich source for discovery of novel genes involved in VLCFA biosynthesis.

To understand the biosynthesis of $n$-alkadiene and triene, Baba et al. have proposed a list of 55 enzymes that are involved in biosynthesis of VLCFA [24]. Nineteen out of the 55 enzymes are found in B. braunii Bot- 88 . In this study, we find 20 out of the 55 enzymes involved in the biosynthesis of the VLCFA, 13 of which are those previously found in $B$. braunii Bot- 88 (i.e., p value $<0.05$ ), consistent with the notion that A race $B$. braunii strains belong to the same sub-species. Eight more enzymes involved in biosynthesis of VLCFA are found in this study. Additionally, 36 out of 100 enzymes in B. braunii Showa [21] involved in biosynthesis of botryococcene or squalene are found in B. braunii 779 (see Fig. 9), consistent with the level of similarity between the two transcriptomes (see Fig. 4).

\section{Conclusions}

Taken together, our results show that the subisolate $B$. braunii 779 exhibits a moderate growth rate (i.e., up to 1.23 gram of CDW per liter per day in $2 \times$ BB medium) and high lipid content (i.e., up to $75 \%$ of DCW after induction by ND), attractive for biofuel production. Comparative transcriptomic analysis between A (i.e., $B$. braunii 779 and Bot-88) and B (i.e., B. braunii Showa, Bot-22, and Bot-70) races indicates that difference races of $B$. braunii strains may belong to different sub-species. Many homologs of unknown sequences found in B. braunii 779 and Bot- 88 suggest that these unknown sequences are most likely novel genes, part of which possibly encodes enzymes involved in VLCFA biosynthesis. This is consistent with the observation of half of the proposed enzymes involved in VLCFA biosynthesis has not been found in A race B. braunii. We propose that $B$. braunii transcriptomes provide a rich source for discovery of novel genes involved in hydrocarbon biosynthesis.

\section{Methods}

\section{Algal culture manipulation}

The algae culture CCALA779 was obtained from the Culture Collection of Autotrophic Organisms, Czech (ccala.butbn.cas.cz) and a subisolate B. braunii 779 was obtained as rapidly growing colonies on Bold's modified Bristol (BB) [30] solid medium. The subisolate was cultivated using $\mathrm{BB}$ (or $2 \times \mathrm{BB}$ ) liquid medium in a $2.5-\mathrm{L}$ low-form flask with shaking at $100 \mathrm{rpm}$ at $25{ }^{\circ} \mathrm{C}$ under continuous illumination flux density of $\sim 250 \mu \mathrm{mol}$ photon $\mathrm{m}^{-2} \mathrm{~s}^{-1}$. Culture was shaken at $100 \mathrm{rpm}$ at $25^{\circ} \mathrm{C}$ (or RT) supplied with $2 \% \mathrm{CO}_{2}$ through bubbling. For analysis of the subisolate in response to ND, the log-phase cells in nitrogen-replete $2 \times \mathrm{BB}$ medium (or $2 \mathrm{BB}+\mathrm{N}$ ) were harvested by centrifugation at 2,000 $\mathrm{rcf}$ at $8{ }^{\circ} \mathrm{C}$ for $5 \mathrm{~min}$ 
and resuspended in nitrogen-depleted $2 \times \mathrm{BB}$ medium (or 2BB-N) isovolumetrically. After growing in 2BB-N medium for 3 days, cells were harvested by filtration for cell dry weight analysis and centrifugation for lipid content and transcriptomic analyses.

For growth rate analysis, cell dry weight (CDW) was determined using the gravimetric methodology. In brief, approximately $200 \mathrm{ml}$ (i.e., for the first 2 time points) or $100 \mathrm{ml}$ (i.e., for the time points at 4 days and after) from the cell culture was harvested by filtration using the glass fiber filter GF/A (Whatman/GE Healthcare, Kent, UK) and dried in oven at $80{ }^{\circ} \mathrm{C}$ overnight. CDW was gravimetrically determined in triplicate using the AG204 balance (Mettler-Toledo Inc., Columbus, OH, USA). Growth rate was estimated using the exponential growth formula

$$
r=\left(\frac{x_{t_{2}}}{x_{t_{1}}}\right)^{1 /\left(t_{2}-t_{1}\right)}-1
$$

where $r$ is growth rate in a time interval from $t_{2}$ to $t_{1}$, and $x_{t_{2}}$ and $x_{t_{1}}$ is the CDW at time points $t_{2}$ and $t_{1}$, respectively. Here, the time interval $t_{2}-t_{1}$ is 2 days. The growth rates at each 2-day interval from day 0 to day 8 are presented in Table 2.

\section{Determination of chlorophyll contents}

To determine chlorophyll content, cells were collected by centrifugation and the cell pellet was resuspended in $96 \%$ ethanol and broken in the glass-bead beater (FastPrep, MP Biomedicals, Solon, OH, USA). After incubation with ethanol for $2 \mathrm{~h}$ at $4{ }^{\circ} \mathrm{C}$, cell debris was removed using centrifugation. The resulting supernatant was subjected to OD measurement at the wavelength of $645 \mathrm{~nm}$ and $663 \mathrm{~nm}$, with the $96 \%$ ethanol as blank. Total chlorophyll content (i.e., chl a and $\mathrm{b}$ ) was estimated using a formula $\mathrm{C}(\mathrm{mg} / \mathrm{L})=20.2 \mathrm{OD}_{645}+8.05 \mathrm{OD}_{663}[46]$ and was converted to percent of CDW.

\section{Determination of photosynthesis efficiency}

To determine photosynthesis efficiency, cells were subjected to PAM-fluorescence analysis using a fluorometer (Imaging-PAM, Heinz Walz GmbH, Effeltrich, Germany) according to the manufacturer's instruction. Briefly, the $\mathrm{Fm}^{\prime}$ and $\mathrm{F}$ were determined at the arctic light of $250 \mu \mathrm{mol}$ photon $\mathrm{m}^{-2} \mathrm{~s}^{-1}$ to mimic the growth conditions. The quantum yield was based on the formula $Y(I I)=\left(\mathrm{Fm}^{\prime}-\mathrm{F}\right) / \mathrm{Fm}^{\prime}[47]$.

\section{Fluorescence microscopic analyses}

To examine the accumulation of lipid and hydrocarbon contents in B. braunii 779 cells, $4 \mu \mathrm{l}$ of $0.25 \mathrm{mg} / \mathrm{ml}$ Nile red in acetone was added to $1 \mathrm{ml}$ of fresh culture according to a previously described method [22]. In brief, after incubation at RT in dark for $15 \mathrm{~min}$, fluorescence signals in the cells were examined using the Zeiss Axiovert $200 \mathrm{M}$ (Carl Zeiss AG, Oberkochen, Germany) with a Zeiss EC Plane Neofluar 40x/0.75 objective lens with a filter cube no. 15 (EX BP 546/12, BS FT 580, EM LP 590). The images were captured by a CoolSNAP HQ monochrome digital camera (Roper Scientific, Ottobrunn, Germany) and processed using MetaMorph software (Molecular Devices, Sunnyvale, CA, USA).

\section{Analysis of lipid and hydrocarbon contents in B. braunii 779 cells}

Total lipids and hydrocarbons were extracted using methanol/chloroform (2:1 by volume) and hexane solutions, respectively. In brief, cell samples taken from cultures at various time points were harvested by centrifugation. The resulting cell pellet was resuspended in $0.5 \mathrm{ml}$ PBS buffer $\left(10 \mathrm{mM} \mathrm{Na} \mathrm{HPO}_{4} / 1.8 \mathrm{mM} \mathrm{K}_{2} \mathrm{PO}_{4} / 137 \mathrm{mM}\right.$ $\mathrm{NaCl} / 2.7 \mathrm{mM} \mathrm{KCl}, \mathrm{pH} 7.4$ ) and mixed with $0.5 \mathrm{~g}$ of the $0.5 \mathrm{~mm}$ acid-washed glass beads (Cat no. G2968, SigmaAldrich, St. Louis, MO, USA). Cells were subsequently broken by 6 bursts using a glass-bead beater (MP Biomedicals). Each burst was set for $30 \mathrm{~s}$ and a 2-min interval was set between bursts. Broken cells were mixed with 10 volumes of methanol/chloroform (2:1 by volume) or hexane overnight. Organic phase was separated from cell debris and glass beads by centrifugation and transferred into a fresh tube. The tube was subsequently placed in a fume hood with a stream of nitrogen gas to evaporate the solvent. The resulting lipids or hydrocarbons were quantified gravimetrically using the AG204 balance (MettlerToledo Inc.).

The lipids were dissolved in chloroform at a concentration of $0.1 \mathrm{mg} / \mu \mathrm{l}$. Equal amount of lipid was loaded on silica TLC plate (60F254, Merck Corporate, Whitehouse Station, NJ, USA) and developed in hexane/diethyl ether/ acetic acid (35:15:0.1 by volume). TAG (tri-oleic acid (C18:1, [cis-9]) glyceride) and FFA (oleic acid (C18:1, [cis-9])) (Sigma-Aldrich, St. Louis, MO, USA) and others were used as standard. Lipid profile on TLC plate was visualized using iodine vapor. The hydrocarbons were dissolved in hexane at a concentration of $0.1 \mathrm{mg} / \mu \mathrm{l}$ and analyzed using Shimadzu GC/MS-QP2010 Plus system (Shimadzu, Kyoto, Japan) equipped with an HP-5 ms Ultra Inert column $(30 \mathrm{~m} \times 0.25 \mathrm{~mm} \times 0.25 \mu \mathrm{m}$, Agilent Technologies, Santa Clara, CA, USA).

\section{S rDNA sequence-based phylogenetic analysis}

Primer sequences (CV1: 5'-TACCTGGTTGATCCTGC CAGTAG-3'; CV2: 5'-CCAATCCCTAGTCGGCATCG T-3'; CV3: 5'-AGATACCGTCGTAGTCTCAACCATA 
A-3'; CV4: 5'-ACCTTGTTACGACTTCTCCTTCCTC-3') used in the study of $18 \mathrm{~S}$ rDNA in several $B$. braunii strains [32] were applied in this study, namely the full length sequence of the $18 \mathrm{~S}$ rDNA were amplified in two fragments: the left fragment of $\sim 1.0 \mathrm{~Kb}$ by primers $\mathrm{CV} 1$ and $\mathrm{CV} 2$ and right fragment of $\sim 0.8 \mathrm{~Kb}$ by primers $\mathrm{CV} 3$ and CV4 (see Additional file 11: Figure S2). Sequences of the left and right fragment were assembled into the full length of $18 \mathrm{~S}$ rDNA of the $B$. braunii 779 with an accession number of BankIt1827606 in NCBI nucleotide database. The $B$. braunii $77918 \mathrm{~S}$ rDNA sequence with others (obtained from treeBASE at http://www.treebase.org) was aligned using ClustalX software with Blosum matrix and the phylogenetic tree was obtained using the Bootstrapped Neighbor Joining tree method $[48,49]$.

\section{Next-generation sequencing analysis}

Total RNA was extracted from B. braunii 779 cells prior to and after ND using TRIzol Plus RNA Purification System (Invitrogen-LifeTechnologies Co., Carlsbad, CA, USA) according to manufacturer's protocol. Approximately $4 \mu \mathrm{g}$ of the resulting total RNA was used for synthesis of cDNA using the TruSeq RNA Sample Prep Kit (Invitrogen-Life Technologies Co.) according to manufacturer's instruction including synthesis of first- and second-strand cDNA, end repair, 3'-end adenylation, adapter ligation, fragment enrichment (e.g., 260 bps in length), and library validation, quantification, and quality assessment with a bioanalyzer (Agilent Technologies; Santa Clara, CA, USA). The libraries are sequenced using the Illumina HiSEQ 2000 Sequencer (BGI, Shenzhen, China).

Over 2 Giga-base clean paired-end reads (90 bps in length/read) from each library were generated using HiSEQ 2000 technology. A total of 55 million reads from both growth conditions were pooled and subjected to de novo assembly using the trinity software [33] (http://trinityrnaseq.github.io) (for detailed description, see Additional file 12). As a result, $\sim 60$ thousand non-redundant contigs/scaffolds/ESTs/cDNA (EST was used in this study for simplicity) were obtained based on a cutoff of EST length $>300$ base pairs and read count $>40$ per EST. To compare transcription levels in different growth conditions, read counts per EST and its normalized level of FPKM (Fragments Per Kilobase per Million mapped reads) were generated using RSEM software [44]. ESTs with at least one 0 FPKM in 4 measurements were filtered out in this analysis. Deferentially transcribed ESTs upon ND is obtained using EdgeR software $[45,50]$ based on the raw counts per EST generated by
RSEM software [44] using a cutoff of fold change $>2$ and $\mathrm{p}$ value $<0.05$.

\section{Annotation of the B. braunii 779 transcriptome}

All non-redundant ESTs were subjected to sequence homology comparison using the Basic Local Aliment Search Tool BLASTX suit against the pool of "best" proteins in 6 comprehensively annotated microalgae genomes: Coccomyxa subellipsoidea C-169 v2 [34], Chlorella variabilis NC64A v1 [35], Chlamydomonas reinhardtiiv4 [36], Micromonas pusilla RCC299 v3 [37], Ostreococcus lucimarinus v2 [38] and Thalassiosira pseudonana CCMP 1335 v3 [39] (genome. jgi-psf.org). A total of 12,292 ESTs showed to share homology to at least a best-hit (i.e., expectation value $<1 \mathrm{E}-07$, length of homology to subject/full length $>40 \%$ ) in the pool of "best" proteins of the 6 genomes. Of 12,292 ESTs, 8,888 (or 72.3\%) and 3,368 (or 27.4\%) were found to be associated with at least one GO function and KEGG ortholog (i.e., expectation value $<1 \mathrm{E}-06$ ), respectively (see Additional file 2 : Table S2, Additional file 4: Table S3).

\section{Pathway-based analysis for potentially co-transcribed subsets of ESTs}

We assumed that a subset of co-regulated ESTs that were associated with a KEGG metabolic pathway or GO biological process would exhibit the coherent expression or similar transcription level (i.e. based on rank by level) in log-phase cells or transcription ratio (i.e., based on rank by ratio) in nitrogen-starved cells. To identify the subset of potentially co-regulated ESTs, we applied a sliding window of 1,024 consecutive ESTs in size and 512 ESTs in step for finding subsets of ESTs of a pathway whose occurrence density is twofold higher than that of background with a p value of less than 0.05 after Bonferroni correction [40, 41].

\section{Statistical analyses}

Binomial test was used to determine the statistical significance of enrichment for pathway-associated ESTs (i.e., success) that are present within a window of ranked ESTs (i.e., trials). Bonferroni correction $[40,41]$ is applied in all multiple tests in this study.

The raw HiSEQ 2000 paired-end sequencing data, the non-redundant transcriptome data, and the annotated transcriptome data generated in this study are available at the NCBI's GEO database (http://www.ncbi.nlm.nih.gov/ geo) with an accession number Series GES71296 (including Platform GPL20730, Samples GSM1831893-96, RSA SPR058734, GES71296 supplemental tables). 


\section{Additional files}

\author{
Additional file 1: Table S1. List of the 12041 annotated ESTs in B. \\ braunii 779 .
}

Additional file 2: Table S2. List of GO terms and number of ESTS associated.

Additional file 3: Figure S1. Ten GO cellular component (CC) and molecular function (MF) categories associated with the most number of ESTs.

Additional file 4: Table S3. List of KEGG pathways and number of ESTS associated.

Additional file 5: Tables S4-7. List of homologous ESTs between $B$. braunii 779 and other strains.

Additional file 6: Tables S8-11. List of homologous ESTs between $B$. braunii Showa and other trains.

Additional file 7: Table S12. List of the differentially transcribed ESTS.

Additional file 8: Table S13. List of B. braunii 779 enzymes involved in VLCFA biosynthesis based on the transcriptome of B. braunii Bot- 88 .

Additional file 9: Table S14. List of B. braunii 779 enzymes involved in botryococcene or squalene biosynthesis based on the curated transcriptome of B. braunii Showa.

Additional file 10: Table S15. List of B. braunii 779 enzymes involved in botryococcene or squalene biosynthesis based on the machine-assembled transcriptome of B. braunii Showa.

Additional file 11: Figure S2. Sequence analysis of the $18 \mathrm{~S}$ rDNA in $B$. braunii 779 .

Additional file 12: Supplemental Methods. Detailed steps of transcriptome analysis.

\section{Abbreviations}

CDW: cell dry weight; FPKM: fragments per kilobase per million mapped reads; GC-MS: gas chromatography-coupled mass spectrometry; ND: nitrogen deprivation; TLC: thin layer chromatography; VLCFA: very long chain fatty acid.

\section{Authors' contributions}

$\mathrm{FL}, \mathrm{DS}, \mathrm{JH}, \mathrm{SQ}$, and WC carried out the biological and biochemical studies; $Z X, X C$, and JL carried out the bioinformatics and statistical studies; WC, and $J \mathrm{~L}$ conceived of the study and participated in its design and coordination; $J$ drafted the manuscript. All authors read and approved the final manuscript.

\section{Author details}

${ }^{1}$ Collaborative Innovation Center of Deep Sea Biology, Ocean College, Zhejiang University, Hangzhou 310058, Zhejiang, China. ${ }^{2}$ Genome Institute of Singapore, A-STAR, Singapore 138672, Singapore. ${ }^{3}$ Ocean Research Centre of Zhoushan, Zhejiang University, 10 Tiyu Road, Room 502, Zhoushan 316021 Zhejiang, China. ${ }^{4}$ Zhejiang Provincial Key Laboratory for Microbial Biochemistry and Metabolic Engineering and College of Life Sciences, Zhejiang University, Hangzhou 310058, Zhejiang, China. ${ }^{5}$ Institute of Chemical and Engineering Sciences, A-STAR, Singapore 627833, Singapore. ${ }^{6}$ Present Address: Dalian Ocean University, Dalian 116023, Liaoning, China. ${ }^{7}$ Present Address: Biopolis Shared Facilities, A-STAR, Singapore 138671, Singapore.

\section{Acknowledgements}

FL was a visiting researcher in the Ocean College, Zhejiang University. This work was initiated in the Genome Institute of Singapore and Institute of Chemical and Engineering Sciences, Singapore by the JCO, A-SATR, Grant (1031C002) to CW and JL and was completed in Zhejiang University, Zhejiang, China by the research grants (2012C31019 and 2014C51020) from the Science and Technology Bureau of the Zhoushan Municipal Government to JL.

\section{Compliance with ethical guidelines}

\section{Competing interests}

The authors declare that they have no competing interests.

Received: 30 May 2015 Accepted: 5 August 2015

Published online: 28 August 2015

\section{References}

1. Wijffels RH, Barbosa MJ (2010) An outlook on microalgal biofuels. Science 329(5993):796-799

2. Rodolfi L, Zittelli GC, Bassi N, Padovani G, Biondi N, Bonini G et al (2009) Microalgae for oil: strain selection, induction of lipid synthesis and out door mass cultivation in a low-cost protobioreactor. Biotechnol Bioeng 102:100-112

3. Singh A, Nigam PS, Murphy JD (2011) Mechanism and challenges in commercialisation of algal biofuels. Bioresour Technol 102(1):26-34

4. Chisti Y (2008) Biodiesel from microalgae beats bioethanol. Trends Biotechnol 26(3):126-131

5. Stephenson AL, Dennis JS, Howe CJ, Scott SA, Smith AG (2010) Influence of nitrogen-limitation regime on the production by Chlorella vulgaris of lipids for biodiesel feedstocks. Biogeosciences. 1:47-58

6. Banerjee A, Sharma R, Chisti Y, Banerjee UC (2002) Botryococcus braunii: a renewable source of hydrocarbons and other chemicals. Crit Rev Biotechnol 22(3):245-279

7. Metzger P, Largeau C (2005) Botryococcus braunii: a rich source for hydrocarbons and related ether lipids. Appl Microbiol Biotechnol 66(5):486-496

8. Niehaus TD, Kinison S, Okada S, Yeo YS, Bell SA, Cui P et al (2012) Functional identification of triterpene methyltransferases from Botryococcus braunii race B. J Biol Chem 287(11):8163-8173

9. Niehaus TD, Okada S, Devarenne TP, Watt DS, Sviripa V, Chappell J (2011) Identification of unique mechanisms for triterpene biosynthesis in Botryococcus braunii. Proc Natl Acad Sci USA 108(30):12260-12265

10. Matsushima D, Jenke-Kodama H, Sato Y, Fukunaga Y, Sumimoto K, Kuzuyama T et al (2012) The single cellular green microalga Botryococcus braunii, race B possesses three distinct 1-deoxy-D-xylulose 5-phosphate synthases. Plant Sci 185-186:309-320

11. Uchida H, Sumimoto K, Ferriols VM, Imou K, Saga K, Furuhashi K et al (2015) Isolation and characterization of two squalene epoxidase genes from Botryococcus braunii, Race B. PLoS One 10(4):e0122649

12. Kagiwada S, Sugita S, Masaike Y, Kato S, Noguchi T (2005) Characterization of coat proteins of COPI- and clathrin-coated vesicles in the unicellular green alga Botryococcus braunii. Plant Sci 169:668-679

13. Metzger P, Villareal-Rosales E, Casadevall E, Couté A (1989) Hydrocarbons, aldehydes and triacylglycerols in some strains of the race $A$ of the green alga Botryococcus braunii. Phytochemistry 28:2349-2353

14. Metzger P, David M, Casadevall E (1987) Biosynthesis of triterpenoid hydrocarbons in the B-race of the green alga Botryococcus braunii. Sites of production and nature of the methylating agent. Phytochemistry 26:129-134

15. Metzger P, Casadevall E (1987) Lycopadiene, a tetraterpenoid hydrocarbon from new strains of the green alga Botryococcus braunii. Tetrahedron Lett 28:3931-3934

16. Okada S, Murakami M, Yamaguchi K (1995) Hydrocarbon composition of newly isolated strains of the green microalga Botryococcus braunii. J Appl Phycol 7:555-559

17. Okada S, Devarenne TP, Murakami M, Abe H, Chappell J (2004) Characterization of botryococcene synthase enzyme activity, a squalene synthaselike activity from the green microalga Botryococcus braunii, Race B. Arch Biochem Biophys 422(1):110-118

18. Okada S, Devarenne TP, Chappell J (2000) Molecular characterization of squalene synthase from the green microalga Botryococcus braunii, race $B$. Arch Biochem Biophys 373(2):307-317

19. Rismani-Yazdi H, Haznedaroglu BZ, Bibby K, Peccia J (2011) Transcriptome sequencing and annotation of the microalgae Dunaliella tertiolecta: 
pathway description and gene discovery for production of next-generation biofuels. BMC Genom 12:148

20. Guarnieri MT, Nag A, Smolinski SL, Darzins A, Seibert M, Pienkos PT (2011) Examination of triacylglycerol biosynthetic pathways via de novo transcriptomic and proteomic analyses in an unsequenced microalga. PLoS One 6(10):e25851

21. Molnar I, Lopez D, Wisecaver JH, Devarenne TP, Weiss TL, Pellegrini M et al (2012) Bio-crude transcriptomics: gene discovery and metabolic network reconstruction for the biosynthesis of the terpenome of the hydrocarbon oil-producing green alga, Botryococcus braunii race B (Showa). BMC Genom 13:576

22. Sun D, Zhu J, Fang L, Zhang X, Chow Y, Liu J (2013) De novo transcriptome profiling uncovers a drastic downregulation of photosynthesis upon nitrogen deprivation in the nonmodel green alga Botryosphaerella sudeticus. BMC Genom 14:715

23. Ioki M, Baba M, Nakajima N, Shiraiwa Y, Watanabe MM (2012) Transcriptome analysis of an oil-rich race B strain of Botryococcus braunii (BOT-70) by de novo assembly of $5^{\prime}$-end sequences of full-length CDNA clones. Bioresour Technol 109:277-281

24. Baba M, loki M, Nakajima N, Shiraiwa Y, Watanabe MM (2012) Transcriptome analysis of an oil-rich race A strain of Botryococcus braunii (BOT$88-2$ ) by de novo assembly of pyrosequencing cDNA reads. Bioresour Technol 109:282-286

25. Ioki M, Baba M, Nakajima N, Shiraiwa Y, Watanabe MM (2012) Transcriptome analysis of an oil-rich race $B$ strain of Botryococcus braunii (BOT-22) by de novo assembly of pyrosequencing cDNA reads. Bioresour Technol 109:292-296

26. Choi GG, Kim BH, Ahn CY, Oh M (2011) Effect of nitrogen limitation on oleic acid biosynthesis in Botryococcus braunii. J Appl Phycol. 23:1031-1037

27. Casadevall E, Dif D, Largeau C, Gudin C, Chaumont D, Desanti O (1985) Studies on batch and continuous cultures of Botryococcus braunii: hydrocarbon production in relation to physiological state, cell structure, and phosphate nutrition. Biotechnol Bioeng 27:286-295

28. Miller R, Wu G, Deshpande RR, Vieler A, Gartner K, Li X et al (2010) Changes in transcript abundance in Chlamydomonas reinhardtii following nitrogen deprivation predict diversion of metabolism. Plant Physiol 154(4):1737-1752

29. Gonzalez-Ballester D, Casero D, Cokus S, Pellegrini M, Merchant SS, Grossman AR (2010) RNA-seq analysis of sulfur-deprived Chlamydomonas cells reveals aspects of acclimation critical for cell survival. Plant Cell 22(6):2058-2084

30. Bold HC (1949) The morphology of Chlamydomonas chlamydogama sp. Bull Torrey Bot Club 76:101-108

31. Greenspan P, Mayer EP, Fowler SD (1985) Nile red: a selective fluorescent stain for intracellular lipid droplets. J Cell Biol 100(3):965-973

32. Senousy HH, Beakes GW, Hack E (2004) Phylogenetic placement of Botryococcus braunii (Trebouxiophyceae) and Botryococcus sudeticus isolate UTEX 2629 (Chlorophyceae). J Phycol 40:412-423

33. Grabherr MG, Haas BJ, Yassour M, Levin JZ, Thompson DA, Amit l et al (2011) Full-length transcriptome assembly from RNA-Seq data without a reference genome. Nat Biotechnol 29(7):644-652
34. Blanc G, Agarkova I, Grimwood J, Kuo A, Brueggeman A, Dunigan DD et al (2012) The genome of the polar eukaryotic microalga Coccomyxa subellipsoidea reveals traits of cold adaptation. Genome Biol 13(5):R39

35. Blanc G, Duncan G, Agarkova I, Borodovsky M, Gurnon J, Kuo A et al (2010) The Chlorella variabilis NC64A genome reveals adaptation to photosymbiosis, coevolution with viruses, and cryptic sex. Plant Cell 22(9):2943-2955

36. Merchant SS, Prochnik SE, Vallon O, Harris EH, Karpowicz SJ, Witman GB et al (2007) The Chlamydomonas genome reveals the evolution of key animal and plant functions. Science 318(5848):245-250

37. Worden AZ, Lee JH, MockT, Rouze P, Simmons MP, Aerts AL et al (2009) Green evolution and dynamic adaptations revealed by genomes of the marine picoeukaryotes Micromonas. Science 324(5924):268-272

38. Palenik B, Grimwood J, Aerts A, Rouze P, Salamov A, Putnam N et al (2007) The tiny eukaryote Ostreococcus provides genomic insights into the paradox of plankton speciation. Proc Natl Acad Sci USA 104(18):7705-7710

39. Armbrust EV, Berges JA, Bowler C, Green BR, Martinez D, Putnam NH et al (2004) The genome of the diatom Thalassiosira pseudonana: ecology, evolution, and metabolism. Science 306(5693):79-86

40. Bland JM, Altman DG (1995) Multiple significance tests: the Bonferroni method. British Med J. 310(6973):170

41. Shi Q, Pavey ES, Carter RE (2012) Bonferroni-based correction factor for multiple, correlated endpoints. Pharm Stat 11(4):300-309

42. Zhang YM, Chen H, He CL, Wang Q (2013) Nitrogen starvation induced oxidative stress in an oil-producing green alga Chlorella sorokiniana C3. PLoS One 8(7):e69225

43. Grant CM (2008) Metabolic reconfiguration is a regulated response to oxidative stress. J Biol 7(1):1

44. Li B, Dewey CN (2011) RSEM: accurate transcript quantification from RNASeq data with or without a reference genome. BMC Bioinf 12:323

45. Blom J, Albaum SP, Doppmeier D, Puhler A, Vorholter FJ, Zakrzewski M et al (2009) EDGAR: a software framework for the comparative analysis of prokaryotic genomes. BMC Bioinf 10:154

46. Sartory DR, Grobbelaar JU (1984) Extraction of chlorophyll a from freshwater phytoplankton for spectrophotometric analysis. Hydrobiologia 114:177-187

47. Klughammer C, Schreibe U (2008) Complementary PS II quantum yields calculated from simple fluorescence parameters measured by PAM fluorometry and the Saturation Pulse method. PAM Appl Notes. 1:27-35

48. Larkin MA, Blackshields G, Brown NP, Chenna R, McGettigan PA, McWilliam H et al (2007) Clustal W and Clustal X version 2.0. Bioinformatics 23(21):2947-2948

49. Thompson JD, Gibson TJ, Plewniak F, Jeanmougin F, Higgins DG (1997) The CLUSTAL_X windows interface: flexible strategies for multiple sequence alignment aided by quality analysis tools. Nucleic Acids Res 25(24):4876-4882

50. Robinson MD, McCarthy DJ, Smyth GK (2010) edgeR: a Bioconductor package for differential expression analysis of digital gene expression data. Bioinformatics 26(1):139-140

\section{Submit your next manuscript to BioMed Central and take full advantage of:}

- Convenient online submission

- Thorough peer review

- No space constraints or color figure charges

- Immediate publication on acceptance

- Inclusion in PubMed, CAS, Scopus and Google Scholar

- Research which is freely available for redistribution

Submit your manuscript at

www.biomedcentral.com/submit
C Biomed Central 